\title{
Global properties of saturated chikungunya virus dynamics models with cellular infection and delays
}

\author{
A.M. Elaiw ${ }^{1 *}\left(\mathbb{D}\right.$, S.E. Almalki ${ }^{1,2}$ and A.D. Hobiny ${ }^{1}$
}

${ }^{*}$ Correspondence:

a_m_elaiw@yahoo.com

${ }^{1}$ Department of Mathematics,

Faculty of Science, King Abdulaziz

University, Jeddah, Saudi Arabia

Full list of author information is

available at the end of the article

\begin{abstract}
This paper studies the global properties of chikungunya virus (CHIKV) dynamics models with both CHIKV-to-monocytes and infected-to-monocyte transmissions. We assume that the infection rate of modeling CHIKV infection is given by saturated mass action. The effect of antibody immune response on the virus dynamics is modeled. The models included three types of time delays, discrete or distributed. The first type of delay is the time between CHIKV entry an uninfected monocyte to be latently infected monocyte. The second time delay is the time between CHIKV entry an uninfected monocyte and the emission of immature CHIKV. The third time delay represents the CHIKV's maturation time. Lyapunov method is utilized and LaSalle's invariance principle is applied to address the global stability of equilibria. The model is numerically simulated to support theoretical results.
\end{abstract}

Keywords: Chikungunya infection; Global stability; Lyapunov function; Time delay; Latency

\section{Introduction}

In the last decades, big efforts have been made to develop and analyze mathematical models for the transmission of the mosquito-borne diseases such as malaria [1-3], dengue [4-6], Zika [7, 8], yellow fever [9], and chikungunya [10-14] to human population. Humans are infected by chikungunya virus (CHIKV) by infected Aedes albopictus and Aedes agypti mosquito. The syndrome of chikungunya includes: fever, headache, severe joint and muscle pain, rash, fatigue, and nausea. The basic mathematical model of within-host virus dynamics was proposed by [15] and has been extended in several works (see, e.g., [16-34]). Wang and Liu [35] have proposed and studied the following within-host CHIKV dynamics model which contains four compartments, uninfected-monocytes $(s)$, infected monocytes $(y)$, free CHIKV particles $(p)$, and antibodies $(x)$ :

$$
\begin{aligned}
& \dot{s}(t)=\varrho-\xi s(t)-\omega_{1} s(t) p(t), \\
& \dot{y}(t)=\omega_{1} s(t) p(t)-\epsilon y(t), \\
& \dot{p}(t)=\varkappa y(t-\delta)-c p(t)-r x(t) p(t), \\
& \dot{x}(t)=\lambda+\rho x\left(t-\delta^{*}\right) p\left(t-\delta^{*}\right)-m x(t) .
\end{aligned}
$$

(c) The Author(s) 2019. This article is distributed under the terms of the Creative Commons Attribution 4.0 International License (http://creativecommons.org/licenses/by/4.0/), which permits unrestricted use, distribution, and reproduction in any medium, provided you give appropriate credit to the original author(s) and the source, provide a link to the Creative Commons license, and indicate if changes were made. 
The susceptible monocytes are generated by rate $\varrho$, die with rate $\xi s(t)$, and are attacked by CHIKV with rate $\omega_{1} s(t) p(t)$. Constants $\epsilon, c$, and $m$ denote, respectively, the normal death rate constants of the infected monocytes, CHIKV, and antibodies. The parameter $\varkappa$ represents the production rate constant of the CHIKV. The term rate $r x(t) p(t)$ represents the neutralization rate of CHIKV. Parameters $\lambda$ and $\rho$ represent the generation and proliferation rate constants of the antibodies. Parameter $\delta$ represents the maturation time of the newly produced CHIKV particles and $\delta^{*}$ represents the time that CHIKV stimulation needs for generating antibodies. Model (1)-(4) presented in [35] has recently been extended in [36, 37]. In [35] and [36, 37] it has been assumed that the uninfected monocyte becomes infected due to its contact with CHIKV (viral infection). It has been mentioned in [38] that the uninfected monocyte can also be infected when it contacts with infected monocyte (cellular infection). Cellular and viral infections have been considered in mathematical models of different viruses in many works (see, e.g., [39-48]).

The main contributions of the paper are summarized as follows:

(1) We incorporate both CHIKV-to-monocyte and infected-to-monocyte transmissions into the CHIKV dynamics model.

(2) The rate of infection in model (1)-(4) is bilinear in the CHIKV and the uninfected monocytes. The actual incidence rate is probably not strictly linear. A less than linear response in CHIKV and infected monocytes could occur due to saturation at high CHIKV or infected monocyte concentrations. Therefore, it is reasonable for us to assume that the infection rate of modeling CHIKV infection is given by saturated mass action.

(3) Latent viral reservoirs serve as a major barrier in curing viral infection. Although antiretroviral treatment reduces the level of viruses significantly, there is still a low viral load due to ongoing latently infected cells reservoir reactivation. Therefore, we propose a model which incorporates two classes of infected monocytes: latently infected monocytes (w), which contain the CHIKV but do not produce it, and actively infected monocytes $(y)$, which produce the CHIKV particles.

(4) In [49], the authors described the CHIKV replication cycle (see Fig. 1): "The virus enters susceptible cells through endocytosis, mediated by an unknown receptor. As the endosome is acidic, conformational changes occur resulting in the fusion of the viral and host cell membranes, causing the release of the nucleocapsid into the cytoplasm. The RNA genome is first translated into the $4 \mathrm{nsPs}$, which together will form the replication complex and assist in several downstream processes (depicted by dashed arrowed line in Fig. 1). Subsequently, the genome is replicated to its negative-sense strand, which in turn will be used as a template for the synthesis of the 49S viral RNA and 26S subgenomic mRNA. The $26 \mathrm{~S}$ subgenomic mRNA will be translated to give the structural proteins ( $\mathrm{C}-\mathrm{pE} 2-$ $6 \mathrm{~K}-\mathrm{E} 1)$. After a round of processing by serine proteases, the capsid is released into the cytoplasm. The remaining structural proteins are further modified post-translationally in the endoplasmic reticulum and subsequently in the Golgi apparatus. E1 and E2 associate as a dimer and are transported to the host plasma membrane, where they will ultimately be incorporated onto the virion surface as trimeric spikes. Capsid protein will form the icosahedral nucleocapsid that will contain the replicated 49S genomic RNA before being assembled into a mature virion ready for budding. During budding the virions will acquire a membrane bilayer from part of the host cell membrane." 


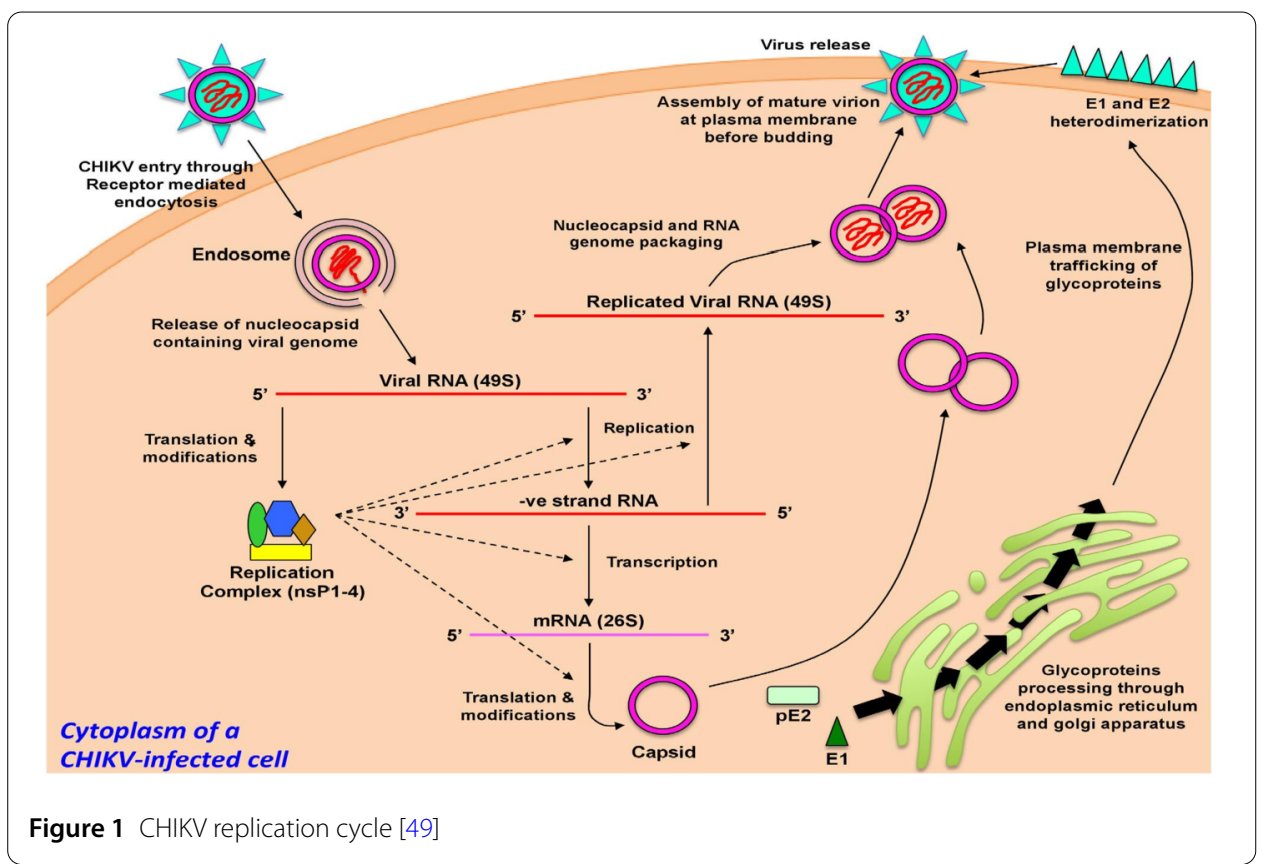

Therefore, this process may take time period which can be incorporated into the CHIKV model by considering three types of discrete or distributed time delays and latently infected cells.

(5) We study the basic and global properties of the proposed models.

We mention that the qualitative analysis presented in this paper is not simply a straightforward generalization of analogous calculations developed in [36] and [37] where only CHIKV-to-monocyte has been considered. This is due to the incorporation of both CHIKV-to-monocyte and infected-to-monocyte transmissions in the present models.

\section{CHIKV model with discrete-delays}

In this section we propose the following CHIKV dynamics model with three discrete-time delays and with both CHIKV-to-monocyte and infected-to-monocyte transmissions:

$$
\begin{aligned}
& \dot{s}(t)=\varrho-\xi s(t)-\frac{\omega_{1} s(t) p(t)}{1+\mu_{1} p(t)}-\frac{\omega_{2} s(t) y(t)}{1+\mu_{2} y(t)} \\
& \dot{w}(t)=(1-n) e^{-a_{1} \delta_{1}}\left[\frac{\omega_{1} s\left(t-\delta_{1}\right) p\left(t-\delta_{1}\right)}{1+\mu_{1} p\left(t-\delta_{1}\right)}+\frac{\omega_{2} s\left(t-\delta_{2}\right) y\left(t-\delta_{1}\right)}{1+\mu_{2} y\left(t-\delta_{1}\right)}\right]-(b+d) w(t), \\
& \dot{y}(t)=n e^{-a_{2} \delta_{2}}\left[\frac{\omega_{1} s\left(t-\delta_{2}\right) p\left(t-\delta_{2}\right)}{1+\mu_{1} p\left(t-\delta_{2}\right)}+\frac{\omega_{2} s\left(t-\delta_{2}\right) y\left(t-\delta_{2}\right)}{1+\mu_{2} y\left(t-\delta_{2}\right)}\right]+d w(t)-\epsilon y(t), \\
& \dot{p}(t)=\varkappa e^{-a_{3} \delta_{3}} y\left(t-\delta_{3}\right)-c p(t)-r x(t) p(t), \\
& \dot{x}(t)=\lambda+\rho x(t) p(t)-m x(t),
\end{aligned}
$$

where $w$ is the concentration of latently infected monocytes. The CHIKV-uninfected and infected-uninfected incidence rates are given by $\frac{\omega_{1} p s}{1+\mu_{1} p}$ and $\frac{\omega_{2} y s}{1+\mu_{2} y}$, where a fraction $n$ (where $0<n<1)$ will become actively infected monocyte and $(1-n)$ will become latently infected monocytes. Constant $b$ denotes the normal death rate constant of the latently infected monocytes. The latently infected monocytes are activated at rate $d w(t)$. Here, $\delta_{1}$ and $\delta_{2}$ 
are the times between CHIKV entry a susceptible monocyte to be latently and actively infected monocytes, respectively. The immature CHIKV needs time $\delta_{3}$ to be mature. The factors $e^{-a_{1} \delta_{1}}, e^{-a_{2} \delta_{2}}$, and $e^{-a_{3} \delta_{3}}$ represent the probability of surviving to the age of $\delta_{1}, \delta_{2}$, and $\delta_{3}$, respectively. Here, $a_{1}, a_{2}$, and $a_{3}$ are positive constants.

We consider the initial conditions

$$
\begin{aligned}
& s(\varphi)=\vartheta_{1}(\varphi), \quad w(\varphi)=\vartheta_{2}(\varphi), \quad y(\varphi)=\vartheta_{3}(\varphi), \\
& p(\varphi)=\vartheta_{4}(\varphi), \quad x(\varphi)=\vartheta_{5}(\varphi), \\
& \vartheta_{i}(\varphi) \geq 0, \quad \varphi \in[-k, 0] \quad \text { and } \quad \vartheta_{i} \in C\left([-k, 0], \mathbb{R}_{\geq 0}\right), \quad i=1,2, \ldots, 5,
\end{aligned}
$$

where $k=\max \left\{\delta_{1}, \delta_{2}, \delta_{3}\right\}$ and $C$ is the Banach space of continuous functions mapping the interval $[-k, 0]$ into $\mathbb{R}_{\geq 0}$.

\subsection{Basic properties}

In the next lemma we establish that the solutions of the system are nonnegative and ultimately bounded.

Lemma 1 The solutions of system (5)-(9) with the initial (10) are nonnegative and ultimately bounded.

Proof Equations (5) and (9) give $\left.\dot{s}\right|_{s=0}=\varrho>0,\left.\dot{x}\right|_{x=0}=\lambda>0$. Thus, for all $t \geq 0, s(t)>0$, and $x(t)>0$. Moreover, from Eqs. (6)-(8), we get

$$
\begin{aligned}
& w(t)=\vartheta_{2}(0) e^{-(b+d) t}+(1-n) e^{-a_{1} \delta_{1}} \int_{0}^{t} e^{-(b+d)(t-\theta)} s\left(\theta-\delta_{1}\right) \\
& \times\left[\frac{\omega_{1} p\left(\theta-\delta_{1}\right)}{1+\mu_{1} p\left(\theta-\delta_{1}\right)}+\frac{\omega_{2} y\left(\theta-\delta_{1}\right)}{1+\mu_{2} y\left(\theta-\delta_{1}\right)}\right] d \theta \geq 0, \\
& y(t)=\vartheta_{3}(0) e^{-\epsilon t}+n e^{-a_{2} \delta_{2}} \int_{0}^{t} e^{-\epsilon(t-\theta)} \\
& \times\left[s\left(\theta-\delta_{2}\right)\left(\frac{\omega_{1} p\left(\theta-\delta_{2}\right)}{1+\mu_{1} p\left(\theta-\delta_{2}\right)}+\frac{\omega_{2} y\left(\theta-\delta_{2}\right)}{1+\mu_{2} y\left(\theta-\delta_{2}\right)}\right)+d w(\theta)\right] d \theta \geq 0, \\
& p(t)=\vartheta_{4}(0) e^{-\int_{0}^{t}(c+r x(\varphi)) d \varphi}+\int_{0}^{t} e^{-\int_{\theta}^{t}(c+r x(\varphi)) d \varphi}\left\{\varkappa e^{-a_{3} \delta_{3}} y\left(\theta-\delta_{3}\right)\right\} d \theta \geq 0 \quad \text { for } t \in[0, k] .
\end{aligned}
$$

By recursive argument, we show that $w(t) \geq 0, y(t) \geq 0$, and $p(t) \geq 0$ for all $t \geq 0$. From Eq. (5) we have $\lim _{t \rightarrow \infty} \sup s(t) \leq \frac{\varrho}{\xi}$. Let us consider

$$
h_{1}(t)=(1-n) e^{-a_{1} \delta_{1}} s\left(t-\delta_{1}\right)+n e^{-a_{2} \delta_{2}} s\left(t-\delta_{2}\right)+w(t)+y(t),
$$

then

$$
\begin{aligned}
\dot{h}_{1}(t)= & (1-n) e^{-a_{1} \delta_{1}}\left[\varrho-\xi s\left(t-\delta_{1}\right)-\frac{\omega_{1} s\left(t-\delta_{1}\right) p\left(t-\delta_{1}\right)}{1+\mu_{1} p\left(t-\delta_{1}\right)}-\frac{\omega_{2} s\left(t-\delta_{1}\right) y\left(t-\delta_{1}\right)}{1+\mu_{2} y\left(t-\delta_{1}\right)}\right] \\
& +n e^{-a_{2} \delta_{2}}\left[\varrho-\xi s\left(t-\delta_{2}\right)-\frac{\omega_{1} s\left(t-\delta_{2}\right) p\left(t-\delta_{2}\right)}{1+\mu_{1} p\left(t-\delta_{2}\right)}-\frac{\omega_{2} s\left(t-\delta_{2}\right) y\left(t-\delta_{2}\right)}{1+\mu_{2} y\left(t-\delta_{2}\right)}\right] \\
& +(1-n) e^{-a_{1} \delta_{1}}\left[\frac{\omega_{1} s\left(t-\delta_{1}\right) p\left(t-\delta_{1}\right)}{1+\mu_{1} p\left(t-\delta_{1}\right)}+\frac{\omega_{2} s\left(t-\delta_{1}\right) y\left(t-\delta_{1}\right)}{1+\mu_{2} y\left(t-\delta_{1}\right)}\right]-(b+d) w(t)
\end{aligned}
$$




$$
\begin{aligned}
& +n e^{-a_{2} \delta_{2}}\left[\frac{\omega_{1} s\left(t-\delta_{2}\right) p\left(t-\delta_{2}\right)}{1+\mu_{1} p\left(t-\delta_{2}\right)}+\frac{\omega_{2} s\left(t-\delta_{2}\right) y\left(t-\delta_{2}\right)}{1+\mu_{2} y\left(t-\delta_{2}\right)}\right]+d w(t)-\epsilon y(t) \\
= & \varrho(1-n) e^{-a_{1} \delta_{1}}+\varrho n e^{-a_{2} \delta_{2}}-(1-n) e^{-a_{1} \delta_{1}} \xi s\left(t-\delta_{1}\right)-n e^{-a_{2} \delta_{2}} \xi s\left(t-\delta_{2}\right) \\
& -b w(t)-\epsilon y(t) \\
\leq & \varrho-\sigma_{1}\left[(1-n) e^{-a_{1} \delta_{1}} s\left(t-\delta_{1}\right)+n e^{-a_{2} \delta_{2}} s\left(t-\delta_{2}\right)+w(t)+y(t)\right] \\
= & \varrho-\sigma_{1} h_{1}(t)
\end{aligned}
$$

where $\sigma_{1}=\min \{\xi, b, \epsilon\}$. It follows that $\lim _{t \rightarrow \infty} \sup h_{1}(t) \leq \Delta_{1}$, where $\Delta_{1}=\frac{\varrho}{\sigma_{1}}$. This yields $\lim _{t \rightarrow \infty} \sup w(t) \leq \Delta_{1}$ and $\lim _{t \rightarrow \infty} \sup y(t) \leq \Delta_{1}$. Define

$$
h_{2}(t)=p(t)+\frac{r}{\rho} x(t)
$$

then

$$
\begin{aligned}
\dot{h}_{2}(t) & =\varkappa e^{-a_{3} \delta_{3}} y\left(t-\delta_{3}\right)-c p(t)+\frac{r}{\rho} \lambda-\frac{r}{\rho} m x(t) \\
& \leq \varkappa \Delta_{1}+\frac{r}{\rho} \lambda-\sigma_{2}\left[p(t)+\frac{r}{\rho} x(t)\right] \\
& =\frac{\varkappa \rho \Delta_{1}+r \lambda}{\rho}-\sigma_{2} h_{2}(t),
\end{aligned}
$$

where $\sigma_{2}=\min \{c, m\}$. Then $\lim _{t \rightarrow \infty} \sup h_{2}(t) \leq \Delta_{2}$, where $\Delta_{2}=\frac{\varkappa \rho \Delta_{1}+r \lambda}{\rho \sigma_{2}}$. The nonnegativity of the solution implies that $\lim _{t \rightarrow \infty} \sup p(t) \leq \Delta_{2}$ and $\lim _{t \rightarrow \infty} \sup x(t) \leq \Delta_{3}$, where $\Delta_{3}=\frac{\rho}{r} \Delta_{2}$. This shows the ultimate boundedness of $s(t), w(t), y(t), p(t)$, and $x(t)$.

Proving the nonnegativity and boundedness of solutions of the model assures the biological viability of the model; and so, we now move on to determining the equilibria of the system. So, in the next section, the threshold parameter for the existence of equilibria is calculated.

\subsection{Equilibria}

We define the basic reproduction number of model (5)-(9) as follows:

$$
\mathcal{R}_{0}=\frac{\alpha_{1} \varrho\left[m \varkappa \omega_{1} e^{-a_{3} \delta_{3}}+\omega_{2}(c m+r \lambda)\right]}{\xi \epsilon(c m+r \lambda)},
$$

where $\alpha_{1}=\frac{d(1-n) e^{-a_{1} \delta_{1}+n(b+d) e^{-a_{2} \delta_{2}}}}{b+d}$. The parameter $R_{0}$ represents the average number of secondary infections and it can be written as $R_{0}=R_{01}+R_{02}$, where

$$
\begin{aligned}
& \mathcal{R}_{01}=\frac{\alpha_{1} \varrho m \varkappa \omega_{1} e^{-a_{3} \delta_{3}}}{\xi \epsilon(c m+r \lambda)}, \\
& \mathcal{R}_{02}=\frac{\alpha_{1} \varrho \omega_{2}}{\xi \epsilon} .
\end{aligned}
$$

In fact, $R_{01}$ is the average number of secondary viruses caused by a virus, that is, the basic reproduction number corresponding to CHIKV-to-monocyte infection mode, while $R_{02}$ 
is the average number of secondary infected monocytes caused by an infected monocyte, that is, the basic reproduction number corresponding to infected-to-monocyte infection mode.

Remark 1 We note that the basic reproduction number of model (1)-(4) presented in [35] is given by

$$
\mathcal{R}_{(1)-(4)}=\frac{\varrho m \varkappa \omega_{1}}{\xi \epsilon(c m+r \lambda)},
$$

which does not depend on the maturation time delay $\delta$. Therefore, the time delay $\delta$ does not affect the stability properties of the equilibria. In contrast, since the basic reproduction number $R$ of our proposed model depends on time delay parameters $\delta_{1}, \delta_{2}$, and $\delta_{3}$, the time delay has significant effect on the stability properties of the equilibria.

Lemma 2 If $\mathcal{R}_{0} \leq 1$, then system (5)-(9) has only one equilibrium $\Omega_{0}$, and if $\mathcal{R}_{0}>1$, then the system has two equilibria $\Omega_{0}$ and $\Omega_{1}$.

The proof of Lemma 2 is given in the Appendix.

Remark 2 In the proof of Lemma 1, we follow the same lines as the proof of Theorem 2.1 in [35]. However, in [35], the existence of equilibria depended on the roots of quadratic equation

$$
q_{1} p^{2}+q_{2} p+q_{3}=0
$$

which is simpler than equation (12). This difference comes from the incorporation of infected-to-monocyte transmission in our model.

\subsection{Global properties}

The global stability of the equilibria will be established by constructing Lyapunov functions following the method presented [50] and followed by [51-56]. To construct the required Lyapunov functional, a positive definite function

$$
G(z)=z-1-\ln z
$$

has been used. It can be easily proved that $G(z) \geq 0$ for any $z>0$. We use the notation $(s, w, y, p, x)=(s(t), w(t), y(t), p(t), x(t))$. Now the following two theorems are stated, respectively, for the global stability of $\Omega_{0}$ and $\Omega_{1}$.

Theorem 1 For system (5)-(9), if $\mathcal{R}_{0} \leq 1$, then $\Omega_{0}$ is globally asymptotically stable (G.A.S.).

Proof Construct a Lyapunov function $U_{0}(s, w, y, p, x)$ as follows:

$$
\begin{aligned}
U_{0}= & \alpha_{1} s_{0} G\left(\frac{s}{s_{0}}\right)+\frac{d}{b+d} w+y+\frac{\alpha_{1} \omega_{1} s_{0}}{c+r x_{0}} p+\frac{r \alpha_{1} \omega_{1} s_{0}}{\rho\left(c+r x_{0}\right)} x_{0} G\left(\frac{x}{x_{0}}\right) \\
& +\frac{d(1-n) e^{-a_{1} \delta_{1}}}{b+d} \int_{0}^{\delta_{1}}\left(\frac{\omega_{1} s(t-\theta) p(t-\theta)}{1+\mu_{1} p(t-\theta)}+\frac{\omega_{2} s(t-\theta) y(t-\theta)}{1+\mu_{2} y(t-\theta)}\right) d \theta
\end{aligned}
$$




$$
\begin{aligned}
& +n e^{-a_{2} \delta_{2}} \int_{0}^{\delta_{2}}\left(\frac{\omega_{1} s(t-\theta) p(t-\theta)}{1+\mu_{1} p(t-\theta)}+\frac{\omega_{2} s(t-\theta) y(t-\theta)}{1+\mu_{2} y(t-\theta)}\right) d \theta \\
& +\frac{\alpha_{1} \omega_{1} s_{0} \varkappa e^{-a_{3} \delta_{3}}}{c+r x_{0}} \int_{0}^{\delta_{3}} y(t-\theta) d \theta .
\end{aligned}
$$

Clearly, $U_{0}\left(s_{0}, 0,0,0, x_{0}\right)=0$ and $U_{0}(s, w, y, p, x)>0$ for all $s, y, p, x>0$. Calculating $\frac{d U_{0}}{d t}$ along system (5)-(9), we obtain

$$
\begin{aligned}
\frac{d U_{0}}{d t}= & \alpha_{1}\left(1-\frac{s_{0}}{s}\right)\left(\varrho-\xi s-\frac{\omega_{1} s p}{1+\mu_{1} p}-\frac{\omega_{2} s y}{1+\mu_{2} y}\right) \\
& +\frac{d}{b+d}\left[(1-n) e^{-a_{1} \delta_{1}} s\left(t-\delta_{1}\right)\left(\frac{\omega_{1} p\left(t-\delta_{1}\right)}{1+\mu_{1} p\left(t-\delta_{1}\right)}+\frac{\omega_{2} y\left(t-\delta_{1}\right)}{1+\mu_{2} y\left(t-\delta_{1}\right)}\right)-(b+d) w\right] \\
& +n e^{-a_{2} \delta_{2}} s\left(t-\delta_{2}\right)\left(\frac{\omega_{1} p\left(t-\delta_{2}\right)}{1+\mu_{1} p\left(t-\delta_{2}\right)}+\frac{\omega_{2} y\left(t-\delta_{2}\right)}{1+\mu_{2} y\left(t-\delta_{2}\right)}\right)+d w-\epsilon y \\
& +\frac{\alpha_{1} \omega_{1} s_{0}}{c+r x_{0}}\left(\varkappa e^{-a_{3} \delta_{3}} y\left(t-\delta_{3}\right)-c p-r x p\right)+\frac{r \alpha_{1} \omega_{1} s_{0}}{\rho\left(c+r x_{0}\right)}\left(1-\frac{x_{0}}{x}\right)(\lambda+\rho x p-m x) \\
& +\frac{d(1-n) e^{-a_{1} \delta_{1}}}{b+d}\left[\frac{\omega_{1} s p}{1+\mu_{1} p}+\frac{\omega_{2} s y}{1+\mu_{2} y}\right) \\
& \left.-\left(\frac{\omega_{1} s\left(t-\delta_{1}\right) p\left(t-\delta_{1}\right)}{1+\mu_{1} p\left(t-\delta_{1}\right)}+\frac{\omega_{2} s\left(t-\delta_{1}\right) y\left(t-\delta_{1}\right)}{1+\mu_{2} y\left(t-\delta_{1}\right)}\right)\right] \\
& +n e^{-a_{2} \delta_{2}}\left[\frac{\omega_{1} s p}{1+\mu_{1} p}+\frac{\omega_{2} s y}{1+\mu_{2} y}-\left(\frac{\omega_{1} s\left(t-\delta_{2}\right) p\left(t-\delta_{2}\right)}{1+\mu_{1} p\left(t-\delta_{2}\right)}+\frac{\omega_{2} s\left(t-\delta_{2}\right) y\left(t-\delta_{2}\right)}{1+\mu_{2} y\left(t-\delta_{2}\right)}\right)\right] \\
& +\frac{\alpha_{1} \omega_{1} s_{0}}{c+r x_{0}} \varkappa e^{-a_{3} \delta_{3}}\left(y-y\left(t-\delta_{3}\right)\right) .
\end{aligned}
$$

Simplify Eq. (13) as follows:

$$
\begin{aligned}
\frac{d U_{0}}{d t}= & -\alpha_{1} \xi \frac{\left(s-s_{0}\right)^{2}}{s}-\frac{\alpha_{1} \omega_{1} s_{0} \mu_{1} p^{2}}{1+\mu_{1} p}-\frac{\alpha_{1} \omega_{2} s_{0} \mu_{2} y^{2}}{1+\mu_{2} y} \\
& +\epsilon\left(\frac{\alpha_{1} \omega_{2} s_{0}}{\epsilon}+\frac{\alpha_{1} \omega_{1} s_{0} \varkappa e^{-a \delta_{3}}}{\epsilon\left(c+r x_{0}\right)}-1\right) y+\frac{r \alpha_{1} \omega_{1} s_{0}}{\rho\left(c+r x_{0}\right)}\left(1-\frac{x_{0}}{x}\right)\left(m x_{0}-m x\right) \\
= & -\alpha_{1} \xi \frac{\left(s-s_{0}\right)^{2}}{s}-\frac{\alpha_{1} \omega_{1} s_{0} \mu_{1} p^{2}}{1+\mu_{1} p}-\frac{\alpha_{1} \omega_{2} s_{0} \mu_{2} y^{2}}{1+\mu_{2} y}-\frac{r \alpha_{1} \omega_{1} s_{0} m}{\rho\left(c+r x_{0}\right)} \frac{\left(x-x_{0}\right)^{2}}{x} \\
& +\epsilon\left(\mathcal{R}_{0}-1\right) y .
\end{aligned}
$$

If $\mathcal{R}_{0} \leq 1$, then $\frac{d U_{0}}{d t} \leq 0$ for all $s, w, y, p, x>0$. Moreover, $\frac{d U_{0}}{d t}=0$ when $s=s_{0}, x=x_{0}$ and $y=p=0$. Let $D=\left\{(s, w, y, p, x): \frac{d U_{0}}{d t}=0\right\}$ and $N$ be the largest invariant subset of $D$. The trajectory of model (5)-(9) tends to $N$ [57]. All the elements of $N$ satisfy $y=p=0$. Then, from Eq. (7), we get

$$
\dot{y}(t)=0=d w(t) \quad \Longrightarrow \quad w(t)=0
$$

Hence, $N=\left\{\Omega_{0}\right\}$. Therefore by LaSalle's invariance principle for delay systems (Theorem 5.3 of Kuang [58]), we get $\Omega_{0}$ is G.A.S. when $R_{0} \leq 1$.

Theorem 2 For system (5)-(9), if $\mathcal{R}_{0}>1$, then $\Omega_{1}$ is G.A.S. 
Proof Let a function $U_{1}(s, w, y, p, x)$ be defined as follows:

$$
\begin{aligned}
& U_{1}=\alpha_{1} s_{1} G\left(\frac{s}{s_{1}}\right)+\frac{d}{b+d} w_{1} G\left(\frac{w}{w_{1}}\right)+y_{1} G\left(\frac{y}{y_{1}}\right) \\
& +\frac{\alpha_{1} \omega_{1} s_{1} p_{1}}{\varkappa e^{-a_{3} \delta_{3}}\left(1+\mu_{1} p_{1}\right) y_{1}} p_{1} G\left(\frac{p}{p_{1}}\right)+\frac{r \alpha_{1} \omega_{1} s_{1} p_{1}}{\rho \varkappa e^{-a_{3} \delta_{3}}\left(1+\mu_{1} p_{1}\right) y_{1}} x_{1} G\left(\frac{x}{x_{1}}\right) \\
& +\frac{d(1-n) e^{-a_{1} \delta_{1}}}{b+d} \frac{\omega_{1} s_{1} p_{1}}{1+\mu_{1} p_{1}} \int_{0}^{\delta_{1}} G\left(\frac{s(t-\theta) p(t-\theta)\left(1+\mu_{1} p_{1}\right)}{s_{1} p_{1}\left(1+\mu_{1} p(t-\theta)\right)}\right) d \theta \\
& +\frac{d(1-n) e^{-a_{1} \delta_{1}}}{b+d} \frac{\omega_{2} s_{1} y_{1}}{1+\mu_{2} y_{1}} \int_{0}^{\delta_{1}} G\left(\frac{s(t-\theta) y(t-\theta)\left(1+\mu_{2} y_{1}\right)}{s_{1} y_{1}\left(1+\mu_{2} y(\delta-\theta)\right)}\right) d \theta \\
& +n e^{-a_{2} \delta_{2}} \frac{\omega_{1} s_{1} p_{1}}{1+\mu_{1} p_{1}} \int_{0}^{\delta_{2}} G\left(\frac{s(t-\theta) p(t-\theta)\left(1+\mu_{1} p_{1}\right)}{s_{1} p_{1}\left(1+\mu_{1} p(\delta-\theta)\right)}\right) d \theta \\
& +n e^{-a_{2} \delta_{2}} \frac{\omega_{2} s_{1} y_{1}}{1+\mu_{2} y_{1}} \int_{0}^{\delta_{2}} G\left(\frac{s(t-\theta) y(t-\theta)\left(1+\mu_{2} y_{2}\right)}{s_{1} y_{1}\left(1+\mu_{2} y(\delta-\theta)\right)}\right) d \theta \\
& +\frac{\alpha_{1} \omega_{1} s_{1} p_{1}}{1+\mu_{1} p_{1}} \int_{0}^{\delta_{3}} G\left(\frac{y(t-\theta)}{y_{1}}\right) d \theta \text {. }
\end{aligned}
$$

Clearly, $U_{1}(s, w, y, p, x)>0$ for all $s, w, y, p, x>0$ and $U_{1}\left(s_{1}, w_{1}, y_{1}, p_{1}, x_{1}\right)=0$. Calculating $\frac{d U_{1}}{d t}$ along the trajectories of (5)-(9), we obtain

$$
\begin{aligned}
\frac{d U_{1}}{d t}= & \alpha_{1}\left(1-\frac{s_{1}}{s}\right)\left(\varrho-\xi s-\frac{\omega_{1} s p}{1+\mu_{1} p}-\frac{\omega_{2} s y}{1+\mu_{2} y}\right) \\
& +\frac{d}{b+d}\left(1-\frac{w_{1}}{w}\right) \\
& \times\left[(1-n) e^{-a_{1} \delta_{1}}\left(\frac{\omega_{1} s\left(t-\delta_{1}\right) p\left(t-\delta_{1}\right)}{1+\mu_{1} p\left(t-\delta_{1}\right)}+\frac{\omega_{2} s\left(t-\delta_{1}\right) y\left(t-\delta_{1}\right)}{1+\mu_{2} y\left(t-\delta_{1}\right)}\right)-(b+d) w\right] \\
& +\left(1-\frac{y_{1}}{y}\right)\left[n e^{-a_{2} \delta_{2}}\left(\frac{\omega_{1} s\left(t-\delta_{2}\right) p\left(t-\delta_{2}\right)}{1+\mu_{1} p\left(t-\delta_{2}\right)}+\frac{\omega_{2} s\left(t-\delta_{2}\right) y\left(t-\delta_{2}\right)}{1+\mu_{2} y\left(t-\delta_{2}\right)}\right)+d w-\epsilon y\right] \\
& +\frac{\alpha_{1} \omega_{1} s_{1} p_{1}}{\varkappa e^{-a_{3} \delta_{3}}\left(1+\mu_{1} p_{1}\right) y_{1}}\left(1-\frac{p_{1}}{p}\right)\left(\varkappa e^{-a_{3} \delta_{3}} y\left(t-\delta_{3}\right)-c p-r x p\right) \\
& +\frac{r \alpha_{1} \omega_{1} s_{1} p_{1}}{\rho \varkappa e^{-a_{3} \delta_{3}\left(1+\mu_{1} p_{1}\right) y_{1}}\left(1-\frac{x_{1}}{x}\right)\left(\lambda+\rho x p-m_{x}\right)} \\
& +\frac{d(1-n) e^{-a_{1} \delta_{1}}}{b+\omega_{1} s_{1} p_{1}} \frac{s p\left(1+\mu_{1} p_{1}\right)}{1+\mu_{1} p_{1}}-\frac{s\left(t-\delta_{1}\right) p\left(t-\delta_{1}\right)\left(1+\mu_{1} p_{1}\right)}{s_{1} p_{1}\left(1+\mu_{1} p\right)} \\
& +n e_{1}^{-a_{2} \delta_{2}} \frac{\omega_{1} s_{1} p_{1}}{1+\mu_{1} p_{1}}\left[\frac{s p\left(1+\mu_{1} p\left(t-\delta_{1}\right)\right)}{s_{1} p_{1}\left(1+\mu_{1} p\right)}-\frac{s\left(t-\delta_{2}\right) p\left(t-\delta_{2}\right)\left(1+\mu_{1} p_{1}\right)}{s_{1} p_{1}\left(1+\mu_{1} p\left(t-\delta_{2}\right)\right)}\right. \\
& \left.+\ln \left(\frac{s\left(t-\delta_{1}\right) p\left(t-\delta_{1}\right)\left(1+\mu_{1} p\right)}{s p\left(1+\mu_{1} p\left(t-\delta_{1}\right)\right)}\right)\right] \\
& +\frac{d(1-n) e^{-a_{1} \delta_{1}}}{b+\omega_{2} s_{1} y_{1}} \frac{s y\left(1+\mu_{2} y_{1}\right)}{1+\mu_{2} y_{1}}\left[\frac{s\left(t-\delta_{1} y_{1}\left(1+\mu_{2} y\right)\right.}{s p\left(1+\mu_{1} p\left(t-\delta_{2}\right)\right) y\left(t-\delta_{1}\right)\left(1+\mu_{2} y_{1}\right)}\right. \\
s_{1} y_{1}\left(1+\mu_{2} y\left(t-\delta_{1}\right)\right) & s y\left(1+\delta_{2} y\right) \\
& \ln )]
\end{aligned}
$$




$$
\begin{aligned}
& +n e^{-a_{2} \delta_{2}} \frac{\omega_{2} s_{1} y_{1}}{1+\mu_{2} y_{1}}\left[\frac{s y\left(1+\mu_{2} y_{1}\right)}{s_{1} y_{1}\left(1+\mu_{2} y\right)}-\frac{s\left(t-\delta_{2}\right) y\left(t-\delta_{2}\right)\left(1+\mu_{2} y_{1}\right)}{s_{1} y_{1}\left(1+\mu_{2} y\left(t-\delta_{2}\right)\right)}\right. \\
& \left.+\ln \left(\frac{s\left(t-\delta_{2}\right) y\left(t-\delta_{2}\right)\left(1+\mu_{2} y\right)}{s y\left(1+\mu_{2} y\left(t-\delta_{2}\right)\right)}\right)\right] \\
& +\frac{\alpha_{1} \omega_{1} s_{1} p_{1}}{1+\mu_{1} p_{1}}\left[\frac{y}{y_{1}}-\frac{y\left(t-\delta_{3}\right)}{y_{1}}+\ln \left(\frac{y\left(t-\delta_{3}\right)}{y}\right)\right] .
\end{aligned}
$$

Simplifying Eq. (15), we get

$$
\begin{aligned}
& \frac{d U_{1}}{d t}=\alpha_{1}\left(1-\frac{s_{1}}{s}\right)(\varrho-\xi s)+\alpha_{1}\left(\frac{\omega_{1} s_{1} p}{1+\mu_{1} p}+\frac{\omega_{2} s_{1} y}{1+\mu_{2} y}\right) \\
& -\frac{d(1-n) e^{-a_{1} \delta_{1}}}{b+d}\left[\frac{\omega_{1} s\left(t-\delta_{1}\right) p\left(t-\delta_{1}\right)}{1+\mu_{1} p\left(t-\delta_{1}\right)}+\frac{\omega_{2} s\left(t-\delta_{1}\right) y\left(t-\delta_{1}\right)}{1+\mu_{2} y\left(t-\delta_{1}\right)}\right] \frac{w_{1}}{w}+d w_{1} \\
& -n e^{-a_{2} \delta_{2}}\left[\frac{\omega_{1} s\left(t-\delta_{2}\right) p\left(t-\delta_{2}\right)}{1+\mu_{1} p\left(t-\delta_{2}\right)}+\frac{\omega_{2} s\left(t-\delta_{2}\right) y\left(t-\delta_{2}\right)}{1+\mu_{2} y\left(t-\delta_{2}\right)}\right] \frac{y_{1}}{y}-d w \frac{y_{1}}{y}-\epsilon y+\epsilon y_{1} \\
& -\frac{\alpha_{1} \omega_{1} s_{1} p_{1}}{1+\mu_{1} p_{1}} \frac{y\left(t-\delta_{3}\right) p_{1}}{y_{1} p}-\frac{\alpha_{1} \omega_{1} s_{1} p_{1}}{\varkappa e^{-a_{3} \delta_{3}}\left(1+\mu_{1} p_{1}\right) y_{1}} c p+\frac{\alpha_{1} \omega_{1} s_{1} p_{1}}{\varkappa e^{-a_{3} \delta_{3}}\left(1+\mu_{1} p_{1}\right) y_{1}} c p_{1} \\
& +\frac{\alpha_{1} \omega_{1} s_{1} p_{1}}{\varkappa e^{-a_{3} \delta_{3}}\left(1+\mu_{1} p_{1}\right) y_{1}} r x p_{1}-\frac{\alpha_{1} \omega_{1} s_{1} p_{1}}{\varkappa e^{-a_{3} \delta_{3}\left(1+\mu_{1} p_{1}\right) y_{1}}} r x_{1} p \\
& +\frac{r \alpha_{1} \omega_{1} s_{1} p_{1}}{\rho \varkappa e^{-a_{3} \delta_{3}}\left(1+\mu_{1} p_{1}\right) y_{1}}\left(1-\frac{x_{1}}{x}\right)(\lambda-m x) \\
& +\frac{d(1-n) e^{-a_{1} \delta_{1}}}{b+d}\left[\frac{\omega_{1} s_{1} p_{1}}{1+\mu_{1} p_{1}} \ln \left(\frac{s\left(t-\delta_{1}\right) p\left(t-\delta_{1}\right)\left(1+\mu_{1} p\right)}{s p\left(1+\mu_{1} p\left(t-\delta_{1}\right)\right)}\right)\right. \\
& \left.+\frac{\omega_{2} s_{1} y_{1}}{1+\mu_{2} y_{1}} \ln \left(\frac{s\left(t-\delta_{1}\right) y\left(t-\delta_{1}\right)\left(1+\mu_{2} y\right)}{s y\left(1+\mu_{2} y\left(t-\delta_{1}\right)\right)}\right)\right] \\
& +n e^{-a_{2} \delta_{2}}\left[\frac{\omega_{1} s_{1} p_{1}}{1+\mu_{1} p_{1}} \ln \left(\frac{s\left(t-\delta_{2}\right) p\left(t-\delta_{2}\right)\left(1+\mu_{1} p\right)}{s p\left(1+\mu_{1} p\left(t-\delta_{2}\right)\right)}\right)\right. \\
& \left.+\frac{\omega_{2} s_{1} y_{1}}{1+\mu_{2} y_{1}} \ln \left(\frac{s\left(t-\delta_{2}\right) y\left(t-\delta_{2}\right)\left(1+\mu_{2} y\right)}{s y\left(1+\mu_{2} y\left(t-\delta_{2}\right)\right)}\right)\right] \\
& +\frac{\alpha_{1} \omega_{1} s_{1} p_{1}}{1+\mu_{1} p_{1}}\left[\frac{y}{y_{1}}+\ln \left(\frac{y\left(t-\delta_{3}\right)}{y}\right)\right] \text {. }
\end{aligned}
$$

Applying the equilibria conditions for $\Omega_{1}$,

$$
\begin{aligned}
& \varrho=\xi s_{1}+\frac{\omega_{1} s_{1} p_{1}}{1+\mu_{1} p_{1}}+\frac{\omega_{2} s_{1} y_{1}}{1+\mu_{2} y_{1}}, \quad w_{1}=\frac{(1-n) e^{-a_{1} \delta_{1}}}{(b+d)}\left(\frac{\omega_{1} s_{1} p_{1}}{1+\mu_{1} p_{1}}+\frac{\omega_{2} s_{1} y_{1}}{1+\mu_{2} y_{1}}\right) \\
& \epsilon y_{1}=\frac{d(1-n) e^{-a_{1} \delta_{1}}}{b+d}\left(\frac{\omega_{1} s_{1} p_{1}}{1+\mu_{1} p_{1}}+\frac{\omega_{2} s_{1} y_{1}}{1+\mu_{2} y_{1}}\right)+n e^{-a_{2} \delta_{2}}\left(\frac{\omega_{1} s_{1} p_{1}}{1+\mu_{1} p_{1}}+\frac{\omega_{2} s_{1} y_{1}}{1+\mu_{2} y_{1}}\right) \\
& =\alpha_{1}\left(\frac{\omega_{1} s_{1} p_{1}}{1+\mu_{1} p_{1}}+\frac{\omega_{2} s_{1} y_{1}}{1+\mu_{2} y_{1}}\right) \text {, } \\
& c p_{1}=\varkappa e^{-a_{3} \delta_{3}} y_{1}-r x_{1} p_{1}, \quad \lambda=m x_{1}-\rho x_{1} p_{1} \text {, }
\end{aligned}
$$

we get

$$
\frac{d U_{1}}{d t}=\alpha_{1}\left(1-\frac{s_{1}}{s}\right)\left(\xi s_{1}-\xi s\right)+\frac{d(1-n) e^{-a_{1} \delta_{1}}}{b+d} \frac{\omega_{1} s_{1} p_{1}}{1+\mu_{1} p_{1}}\left(1-\frac{s_{1}}{s}\right)
$$




$$
\begin{aligned}
& +n e^{-a_{2} \delta_{2}} \frac{\omega_{1} s_{1} p_{1}}{1+\mu_{1} p_{1}}\left(1-\frac{s_{1}}{s}\right) \\
& +\frac{d(1-n) e^{-a_{1} \delta_{1}}}{b+d} \frac{\omega_{2} s_{1} y_{1}}{1+\mu_{2} y_{1}}\left(1-\frac{s_{1}}{s}\right)+n e^{-a_{2} \delta_{2}} \frac{\omega_{2} s_{1} y_{1}}{1+\mu_{2} y_{1}}\left(1-\frac{s_{1}}{s}\right) \\
& +\frac{\alpha_{1} \omega_{1} s_{1} p_{1}}{1+\mu_{1} p_{1}} \frac{\left(1+\mu_{1} p_{1}\right) p}{\left(1+\mu_{1} p\right) p_{1}}+\frac{\alpha_{1} \omega_{2} s_{1} y_{1}}{1+\mu_{2} y_{1}} \frac{\left(1+\mu_{2} y_{1}\right) y}{\left(1+\mu_{2} y\right) y_{1}} \\
& -\frac{d(1-n) e^{-a_{1} \delta_{1}}}{b+d} \frac{\omega_{1} s_{1} p_{1}}{1+\mu_{1} p_{1}}\left[\frac{s\left(t-\delta_{1}\right) p\left(t-\delta_{1}\right)\left(1+\mu_{1} p_{1}\right) w_{1}}{s_{1} p_{1}\left(1+\mu_{1} p\left(t-\delta_{1}\right)\right) w}\right] \\
& -\frac{d(1-n) e^{-a_{1} \delta_{1}}}{b+d} \frac{\omega_{2} s_{1} y_{1}}{1+\mu_{2} y_{1}}\left[\frac{s\left(t-\delta_{1}\right) y\left(t-\delta_{1}\right)\left(1+\mu_{2} y_{1}\right) w_{1}}{s_{1} y_{1}\left(1+\mu_{2} y\left(t-\delta_{1}\right)\right) w}\right] \\
& +\frac{d(1-n) e^{-a_{1} \delta_{1}}}{b+d}\left[\frac{\omega_{1} s_{1} p_{1}}{1+\mu_{1} p_{1}}+\frac{\omega_{2} s_{1} y_{1}}{1+\mu_{2} y_{1}}\right] \\
& -n e^{-a_{2} \delta_{2}} \frac{\omega_{1} s_{1} p_{1}}{1+\mu_{1} p_{1}}\left[\frac{s\left(t-\delta_{2}\right) p\left(t-\delta_{2}\right)\left(1+\mu_{1} p_{1}\right) y_{1}}{s_{1} p_{1}\left(1+\mu_{1} p\left(t-\delta_{2}\right)\right) y}\right] \\
& -n e^{-a_{2} \delta_{2}} \frac{\omega_{2} s_{1} y_{1}}{1+\mu_{2} y_{1}}\left[\frac{s\left(t-\delta_{2}\right) y\left(t-\delta_{2}\left(1+\mu_{2} y_{1}\right)\right) y_{1}}{s_{1} y_{1}\left(1+\mu_{2} y\left(t-\delta_{2}\right)\right) y}\right] \\
& -\frac{d(1-n) e^{-a_{1} \delta_{1}}}{b+d}\left[\frac{\omega_{1} s_{1} p_{1}}{1+\mu_{1} p_{1}}+\frac{\omega_{2} s_{1} y_{1}}{1+\mu_{2} y_{1}}\right] \frac{w y_{1}}{w_{1} y} \\
& -\frac{\alpha_{1} \omega_{2} s_{1} y_{1}}{1+\mu_{2} y_{1}} \frac{y}{y_{1}}+\frac{d(1-n) e^{-a_{1} \delta_{1}}}{b+d}\left[\frac{\omega_{1} s_{1} p_{1}}{1+\mu_{1} p_{1}}+\frac{\omega_{2} s_{1} y_{1}}{1+\mu_{2} y_{1}}\right] \\
& +n e^{-a_{2} \delta_{2}}\left[\frac{\omega_{1} s_{1} p_{1}}{1+\mu_{1} p_{1}}+\frac{\omega_{2} s_{1} y_{1}}{1+\mu_{2} y_{1}}\right] \\
& -\frac{\alpha_{1} \omega_{1} s_{1} p_{1}}{1+\mu_{1} p_{1}}\left[\frac{y\left(t-\delta_{3}\right) p_{1}}{y_{1} p}\right]-\frac{\alpha_{1} \omega_{1} s_{1} p_{1}}{1+\mu_{1} p_{1}} \frac{p}{p_{1}}+\frac{d(1-n) e^{-a_{1} \delta_{1}}}{b+d} \frac{\omega_{1} s_{1} p_{1}}{1+\mu_{1} p_{1}} \\
& +n e^{-a_{2} \delta_{2}} \frac{\omega_{1} s_{1} p_{1}}{1+\mu_{1} p_{1}} \\
& -\frac{2 r \alpha_{1} s_{1} p_{1} \omega_{1}}{\varkappa e^{-a_{3} \delta_{3}}\left(1+\mu_{1} p_{1}\right) y_{1}} x_{1} p_{1}+\frac{r \alpha_{1} s_{1} p_{1} \omega_{1}}{\varkappa e^{-a_{3} \delta_{3}}\left(1+\mu_{1} p_{1}\right) y_{1}} x_{1} p_{1}\left(\frac{x}{x_{1}}\right) \\
& +\frac{r \alpha_{1} s_{1} p_{1} \omega_{1}}{\varkappa e^{-a_{3} \delta_{3}}\left(1+\mu_{1} p_{1}\right) y_{1}} x_{1} p_{1}\left(\frac{x_{1}}{x}\right) \\
& -\frac{r \alpha_{1} s_{1} p_{1} m \omega_{1}}{\rho \varkappa e^{-a_{3} \delta_{3}}\left(1+\mu_{1} p_{1}\right) y_{1}} \frac{\left(x-x_{1}\right)^{2}}{x} \\
& +\frac{d(1-n) e^{-a_{1} \delta_{1}}}{b+d} \frac{\omega_{1} s_{1} p_{1}}{1+\mu_{1} p_{1}} \ln \left[\frac{s\left(t-\delta_{1}\right) p\left(t-\delta_{1}\right)\left(1+\mu_{1} p\right)}{s p\left(1+\mu_{1} p\left(t-\delta_{1}\right)\right)}\right] \\
& +\frac{d(1-n) e^{-a_{1} \delta_{1}}}{b+d} \frac{\omega_{2} s_{1} y_{1}}{1+\mu_{2} y_{1}} \ln \left[\frac{s\left(t-\delta_{1}\right) y\left(t-\delta_{1}\right)\left(1+\mu_{2} y\right)}{s y\left(1+\mu_{2} y\left(t-\delta_{1}\right)\right)}\right] \\
& +n e^{-a_{2} \delta_{2}} \frac{\omega_{1} s_{1} p_{1}}{1+\mu_{1} p_{1}} \ln \left[\frac{s\left(t-\delta_{2}\right) p\left(t-\delta_{2}\right)\left(1+\mu_{1} p\right)}{s p\left(1+\mu_{1} p\left(t-\delta_{2}\right)\right)}\right] \\
& +n e^{-a_{2} \delta_{2}} \frac{\omega_{2} s_{1} y_{1}}{1+\mu_{2} y_{1}} \ln \left[\frac{s\left(t-\delta_{2}\right) y\left(t-\delta_{2}\right)\left(1+\mu_{2} y\right)}{s y\left(1+\mu_{2} y\left(t-\delta_{2}\right)\right)}\right] \\
& +\frac{\alpha_{1} \omega_{1} s_{1} p_{1}}{1+\mu_{1} p_{1}} \ln \left[\frac{y\left(t-\delta_{3}\right)}{y}\right] \text {. }
\end{aligned}
$$


Using the following equations:

$$
\begin{aligned}
\ln \left(\frac{s\left(t-\delta_{1}\right) p\left(t-\delta_{1}\right)\left(1+\mu_{1} p\right)}{s p\left(1+\mu_{1} p\left(t-\delta_{1}\right)\right)}\right)= & \ln \left(\frac{s_{1}}{s}\right)+\ln \left(\frac{s\left(t-\delta_{1}\right) p\left(t-\delta_{1}\right)\left(1+\mu_{1} p_{1}\right) w_{1}}{s_{1} p_{1}\left(1+\mu_{1} p\left(t-\delta_{1}\right)\right) w}\right) \\
& +\ln \left(\frac{w y_{1}}{w_{1} y}\right)+\ln \left(\frac{y p_{1}}{y_{1} p}\right)+\ln \left(\frac{1+\mu_{1} p}{1+\mu_{1} p_{1}}\right), \\
\ln \left(\frac{s\left(t-\delta_{1}\right) y\left(t-\delta_{1}\right)\left(1+\mu_{2} y\right)}{s y\left(1+\mu_{2} y\left(t-\delta_{1}\right)\right)}\right)= & \ln \left(\frac{s_{1}}{s}\right)+\ln \left(\frac{s\left(t-\delta_{1}\right) y\left(t-\delta_{1}\right)\left(1+\mu_{2} y_{1}\right) w_{1}}{s_{1} y_{1}\left(1+\mu_{2} y\left(t-\delta_{1}\right)\right) w}\right) \\
& +\ln \left(\frac{w y_{1}}{w_{1} y}\right)+\ln \left(\frac{1+\mu_{2} y}{1+\mu_{2} y_{1}}\right), \\
\ln \left(\frac{s\left(t-\delta_{2}\right) p\left(t-\delta_{2}\right)\left(1+\mu_{1} p\right)}{s p\left(1+\mu_{1} p\left(t-\delta_{2}\right)\right)}\right)= & \ln \left(\frac{s_{1}}{s}\right)+\ln \left(\frac{s\left(t-\delta_{2}\right) p\left(t-\delta_{2}\right)\left(1+\mu_{1} p_{1}\right) y_{1}}{s_{1} p_{1}\left(1+\mu_{1} p\left(t-\delta_{2}\right)\right) y}\right) \\
& +\ln \left(\frac{y p_{1}}{y_{1} p}\right)+\ln \left(\frac{1+\mu_{1} p}{1+\mu_{1} p_{1}}\right), \\
\ln \left(\frac{s\left(t-\delta_{2}\right) y\left(t-\delta_{2}\right)\left(1+\mu_{2} y\right)}{s y\left(1+\mu_{2} y\left(t-\delta_{2}\right)\right)}\right)= & \ln \left(\frac{s_{1}}{s}\right)+\ln \left(\frac{s\left(t-\delta_{2}\right) y\left(t-\delta_{2}\right)\left(1+\mu_{2} y_{1}\right)}{s_{1} y\left(1+\mu_{2} y\left(t-\delta_{2}\right)\right)}\right) \\
& +\ln \left(\frac{1+\mu_{2} y}{1+\mu_{2} y_{1}}\right) \\
\ln \left(\frac{y\left(t-\delta_{3}\right)}{y}\right)=\ln \left(\frac{y\left(t-\delta_{3}\right) p_{1}}{y_{1} p}\right)+ & \ln \left(\frac{y_{1} p}{y p_{1}}\right),
\end{aligned}
$$

we get

$$
\begin{aligned}
\frac{d U_{1}}{d t}= & -\alpha_{1} \xi \frac{\left(s-s_{1}\right)^{2}}{s}-\frac{r \alpha_{1} s_{1} p_{1} m \omega_{1}}{\rho \varkappa e^{-a_{3} \delta_{3}}\left(1+\mu_{1} p_{1}\right) y_{1}} \frac{\left(x-x_{1}\right)^{2}}{x} \\
& -\frac{r \alpha_{1} s_{1} p_{1} \omega_{1}}{\varkappa e^{-a_{3} \delta_{3}\left(1+\mu_{1} p_{1}\right) y_{1}} x_{1} p_{1}\left[2-\frac{x}{x_{1}}-\frac{x_{1}}{x}\right]} \\
& +\frac{d(1-n) e^{-a_{1} \delta_{1}}}{b+d}\left(\frac{\omega_{1} s_{1} p_{1}}{1+\mu_{1} p_{1}}+\frac{\omega_{2} s_{1} y_{1}}{1+\mu_{2} y_{1}}\right)\left[1-\frac{s_{1}}{s}+\ln \left(\frac{s_{1}}{s}\right)\right] \\
& +n e^{-a_{2} \delta_{2}}\left(\frac{\omega_{1} s_{1} p_{1}}{1+\mu_{1} p_{1}}+\frac{\omega_{2} s_{1} y_{1}}{1+\mu_{2} y_{1}}\right)\left[1-\frac{s_{1}}{s}+\ln \left(\frac{s_{1}}{s}\right)\right] \\
& +\frac{\alpha_{1} \omega_{1} s_{1} p_{1}}{1+\mu_{1} p_{1}}\left[\frac{\left(1+\mu_{1} p_{1}\right) p}{\left(1+\mu_{1} p\right) p_{1}}-\frac{p}{p_{1}}-1+\frac{1+\mu_{1} p}{1+\mu_{1} p_{1}}\right] \\
& +\frac{\alpha_{1} \omega_{2} s_{1} y_{1}}{1+\mu_{2} y_{1}}\left[\frac{\left(1+\mu_{2} y_{1}\right) y}{\left(1+\mu_{2} y\right) y_{1}}-\frac{y}{y_{1}}-1+\frac{1+\mu_{2} y}{1+\mu_{2} y_{1}}\right] \\
& +\frac{d(1-n) e^{-a_{1} \delta_{1}}}{b+d} \frac{\omega_{1} s_{1} p_{1}}{1+\mu_{1} p_{1}}\left[1-\frac{s\left(t-\delta_{1}\right) p\left(t-\delta_{1}\right)\left(1+\mu_{1} p_{1}\right) w_{1}}{s_{1} p_{1}\left(1+\mu_{1} p\left(t-\delta_{1}\right)\right) w}\right. \\
& \left.+\ln \left(\frac{s\left(t-\delta_{1}\right) p\left(t-\delta_{1}\right)\left(1+\mu_{1} p_{1}\right) w_{1}}{s_{1} p_{1}\left(1+\mu_{1} p\left(t-\delta_{1}\right)\right) w}\right)\right] \\
& +\frac{d(1-n) e^{-a_{1} \delta_{1}}}{b+d} \frac{\omega_{2} s_{1} y_{1}}{1+\mu_{2} y_{1}}\left[1-\frac{s\left(t-\delta_{1}\right) y\left(t-\delta_{1}\right)\left(1+\mu_{2} y_{1}\right) w_{1}}{s_{1} y_{1}\left(1+\mu_{2} y\left(t-\delta_{1}\right)\right) w}\right. \\
& \left.+\ln \left(\frac{s\left(t-\delta_{1}\right) y\left(t-\delta_{1}\right)\left(1+\mu_{2} y_{1}\right) w_{1}}{s_{1} y_{1}\left(1+\mu_{2} y\left(t-\delta_{1}\right)\right) w}\right)\right]
\end{aligned}
$$




$$
\begin{aligned}
& +n e^{-a_{2} \delta_{2}} \frac{\omega_{1} s_{1} p_{1}}{1+\mu_{1} p_{1}}\left[1-\frac{s\left(t-\delta_{2}\right) p\left(t-\delta_{2}\right)\left(1+\mu_{1} p_{1}\right) y_{1}}{s_{1} p_{1}\left(1+\mu_{1} p\left(t-\delta_{2}\right)\right) y}\right. \\
& \left.+\ln \left(\frac{s\left(t-\delta_{2}\right) p\left(t-\delta_{2}\right)\left(1+\mu_{1} p_{1}\right) y_{1}}{s_{1} p_{1}\left(1+\mu_{1} p\left(t-\delta_{2}\right)\right) y}\right)\right] \\
& +n e^{-a_{2} \delta_{2}} \frac{\omega_{2} s_{1} y_{1}}{1+\mu_{2} y_{1}}\left[1-\frac{s\left(t-\delta_{2}\right) y\left(t-\delta_{2}\left(1+\mu_{2} y_{1}\right)\right)}{s_{1}\left(1+\mu_{2} y\left(t-\delta_{2}\right)\right) y}\right. \\
& \left.+\ln \left(\frac{s\left(t-\delta_{2}\right) y\left(t-\delta_{2}\left(1+\mu_{2} y_{1}\right)\right)}{s_{1}\left(1+\mu_{2} y\left(t-\delta_{2}\right)\right) y}\right)\right] \\
& +\frac{d(1-n) e^{-a_{1} \delta_{1}}}{b+d} \frac{\omega_{1} s_{1} p_{1}}{1+\mu_{1} p_{1}}\left[1-\frac{w y_{1}}{w_{1} y}+\ln \left(\frac{w y_{1}}{w_{1} y}\right)\right] \\
& +\frac{d(1-n) e^{-a_{1} \delta_{1}}}{b+\omega_{2} s_{1} y_{1}}\left[1-\frac{w y_{1}}{w_{1} y}+\ln \left(\frac{w y_{1}}{w_{1} y}\right)\right] \\
& +\frac{\alpha_{1} \omega_{1} s_{1} p_{1}}{1+\mu_{1} p_{1}}\left[1-\frac{y\left(t-\delta_{3}\right) p_{1}}{y_{1} p}+\ln \left(\frac{y\left(t-\delta_{3}\right) p_{1}}{y_{1} p}\right)\right] \\
& +\frac{\alpha_{1} \omega_{1} s_{1} p_{1}}{1+\mu_{1} p_{1}}\left[1-\frac{1+\mu_{1} p}{1+\mu_{1} p_{1}}+\ln \left(\frac{1+\mu_{1} p}{1+\mu_{1} p_{1}}\right)\right] \\
& +\frac{\alpha_{1} \omega_{2} s_{1} y_{1}}{1+\mu_{2} y_{1}}\left[1-\frac{1+\mu_{2} y}{1+\mu_{2} y_{1}}+\ln \left(\frac{1+\mu_{2} y}{1+\mu_{2} y_{1}}\right)\right]
\end{aligned}
$$

Finally we get

$$
\begin{aligned}
& \frac{d U_{1}}{d t}=-\alpha_{1} \xi \frac{\left(s-s_{1}\right)^{2}}{s}-\frac{\alpha_{1} r s_{1} p_{1} \omega_{1}}{\rho \varkappa e^{-a_{3} \delta_{3}}\left(1+\mu_{1} p_{1}\right) y_{1}} \frac{\lambda}{x_{1}} \frac{\left(x-x_{1}\right)^{2}}{x} \\
& -\alpha_{1}\left(\frac{\omega_{1} s_{1} p_{1}}{1+\mu_{1} p_{1}}+\frac{\omega_{2} s_{1} y_{1}}{1+\mu_{2} y_{1}}\right) G\left(\frac{s_{1}}{s}\right) \\
& -\frac{\alpha_{1} \omega_{1} s_{1} p_{1}}{1+\mu_{1} p_{1}}\left(\frac{\mu_{1}\left(p-p_{1}\right)^{2}}{\left(1+\mu_{1} p\right)\left(1+\mu_{1} p_{1}\right) p_{1}}\right)-\frac{\alpha_{1} \omega_{2} s_{1} y_{1}}{1+\mu_{2} y_{1}}\left(\frac{\mu_{2}\left(y-y_{1}\right)^{2}}{\left(1+\mu_{2} y\right)\left(1+\mu_{2} y_{1}\right) y_{1}}\right) \\
& -\frac{d(1-n) e^{-a_{1} \delta_{1}}}{b+d} \frac{\omega_{1} s_{1} p_{1}}{1+\mu_{1} p_{1}} G\left(\frac{s\left(t-\delta_{1}\right) p\left(t-\delta_{1}\right)\left(1+\mu_{1} p_{1}\right) w_{1}}{s_{1} p_{1}\left(1+\mu_{1} p\left(t-\delta_{1}\right)\right) w}\right) \\
& -\frac{d(1-n) e^{-a_{1} \delta_{1}}}{b+d} \frac{\omega_{2} s_{1} y_{1}}{1+\mu_{2} y_{1}} G\left(\frac{s\left(t-\delta_{1}\right) y\left(t-\delta_{1}\right)\left(1+\mu_{2} y_{1}\right) w_{1}}{s_{1} y_{1}\left(1+\mu_{2} y\left(t-\delta_{1}\right)\right) w}\right) \\
& -n e^{-a_{2} \delta_{2}} \frac{\omega_{1} s_{1} p_{1}}{1+\mu_{1} p_{1}} G\left(\frac{s\left(t-\delta_{2}\right) p\left(t-\delta_{2}\right)\left(1+\mu_{1} p_{1}\right) y_{1}}{s_{1} p_{1}\left(1+\mu_{1} p\left(t-\delta_{2}\right)\right) y}\right) \\
& -n e^{-a_{2} \delta_{2}} \frac{\omega_{2} s_{1} y_{1}}{1+\mu_{2} y_{1}} G\left(\frac{s\left(t-\delta_{2}\right) y\left(t-\delta_{2}\right)\left(1+\mu_{2} y_{1}\right)}{s_{1}\left(1+\mu_{2} y\left(t-\delta_{2}\right)\right) y}\right) \\
& -\frac{d(1-n) e^{-a_{1} \delta_{1}}}{b+d} \frac{\omega_{1} s_{1} p_{1}}{1+\mu_{1} p_{1}} G\left(\frac{w y_{1}}{w_{1} y}\right)-\frac{d(1-n) e^{-a_{1} \delta_{1}}}{b+d} \frac{\omega_{2} s_{1} y_{1}}{1+\mu_{2} y_{1}} G\left(\frac{w y_{1}}{w_{1} y}\right) \\
& -\frac{\alpha_{1} \omega_{1} s_{1} p_{1}}{1+\mu_{1} p_{1}} G\left(\frac{y\left(t-\delta_{3}\right) p_{1}}{y_{1} p}\right)-\frac{\alpha_{1} \omega_{1} s_{1} p_{1}}{1+\mu_{1} p_{1}} G\left(\frac{1+\mu_{1} p}{1+\mu_{1} p_{1}}\right) \\
& -\frac{\alpha_{1} \omega_{2} s_{1} y_{1}}{1+\mu_{1} y_{1}} G\left(\frac{1+\mu_{1} y}{1+\mu_{1} y_{1}}\right) \text {. }
\end{aligned}
$$

Therefore, $\frac{d U_{1}}{d t} \leq 0$ for all $s, w, y, p, x>0$. Let $\widetilde{D}=\left\{(s, w, y, p, x): \frac{d U_{1}}{d t}=0\right\}$ and $\widetilde{N}$ be the largest invariant subset of $\widetilde{D}$. The trajectory of model (5)-(9) tends to $\widetilde{N}$ [57]. It can be verified 
that $\frac{d U_{1}}{d t}=0$ implies $s=s_{1}, y=y_{1}, p=p_{1}, x=x_{1}$, and

$$
\begin{aligned}
\frac{s\left(t-\delta_{1}\right) p\left(t-\delta_{1}\right)\left(1+\mu_{1} p_{1}\right) w_{1}}{s_{1} p_{1}\left(1+\mu_{1} p\left(t-\delta_{1}\right)\right) w} & =\frac{s\left(t-\delta_{1}\right) y\left(t-\delta_{1}\right)\left(1+\mu_{2} y_{1}\right) w_{1}}{s_{1} y_{1}\left(1+\mu_{2} y\left(t-\delta_{1}\right)\right) w} \\
& =\frac{s\left(t-\delta_{2}\right) p\left(t-\delta_{2}\right)\left(1+\mu_{1} p_{1}\right) y_{1}}{s_{1} p_{1}\left(1+\mu_{1} p\left(t-\delta_{2}\right)\right) y} \\
& =\frac{s\left(t-\delta_{2}\right) y\left(t-\delta_{2}\right)\left(1+\mu_{2} y_{1}\right)}{s_{1}\left(1+\mu_{2} y\left(t-\delta_{2}\right)\right) y}=1 .
\end{aligned}
$$

Applying the above relations to Eq. (6), we get $w(t)=w_{1}$ and hence $\widetilde{N}=\left\{\Omega_{1}\right\}$. This shows that all the sufficient conditions given in Corollary 5.2 of Kuang [58] are satisfied under the condition $R_{0}>1$. This implies that $\Omega_{1}$ is G.A.S. when $R_{0}>1$.

\section{CHIKV model with distributed delays}

In this section, we consider CHIKV dynamics model with distributed delays:

$$
\begin{aligned}
& \dot{s}(t)=\varrho-\xi s(t)-\frac{\omega_{1} s(t) p(t)}{1+\mu_{1} p(t)}-\frac{\omega_{2} s(t) y(t)}{1+\mu_{2} y(t)}, \\
& \dot{w}(t)=(1-n) \int_{0}^{\zeta_{1}} f_{1}(\delta) e^{-a_{1} \delta}\left[\frac{\omega_{1} s(t-\delta) p(t-\delta)}{1+\mu_{1} p(t-\delta)}+\frac{\omega_{2} s(t-\delta) y(t-\delta)}{1+\mu_{2} y(t-\delta)}\right] d \delta \\
&-(b+d) w(t), \\
& \dot{y}(t)=n \int_{0}^{\zeta_{2}} f_{2}(\delta) e^{-a_{2} \delta}\left[\frac{\omega_{1} s(t-\delta) p(t-\delta)}{1+\mu_{1} p(t-\delta)}+\frac{\omega_{2} s(t-\delta) y(t-\delta)}{1+\mu_{2} y(t-\delta)}\right] d \delta+d w(t) \\
&-\epsilon y(t), \\
& \dot{p}(t)= \varkappa \int_{0}^{\zeta_{3}} f_{3}(\delta) e^{-a_{3} \delta} y(t-\delta) d \delta-c p(t)-r x(t) p(t), \\
& \dot{x}(t)=\lambda \rho x(t) p(t)-m x(t),
\end{aligned}
$$

where $f_{i}(\delta)>0, i=1,2,3$, are probability distribution functions satisfying $f_{i}(\delta)>0, i=$ $1,2,3$, and

$$
\int_{0}^{\zeta_{i}} f_{i}(\delta) d \delta=1, \quad \int_{0}^{\zeta_{i}} f_{i}(u) e^{l u} d u<\infty, \quad i=1,2,3
$$

where $l>0$. Denote $F_{i}=\int_{0}^{\zeta_{i}} f_{i}(\delta) e^{-a_{i} \delta} d \delta, i=1,2,3$, thus $0<F_{i} \leq 1$. The initial conditions for system (17)-(21) are given by Eq. (10) with $k=\max \left\{\zeta_{1}, \zeta_{2}, \zeta_{3}\right\}$.

\subsection{Basic properties}

Lemma 3 The solutions of system (17)-(21) with the initial (10) are nonnegative and ultimately bounded. 
Proof Equations (17) and (21) give $\left.\dot{s}\right|_{s=0}=\varrho>0,\left.\dot{x}\right|_{x=0}=\lambda>0$. Thus, for all $t \geq 0 s(t)>0$ and $x(t)>0$. Moreover, from Eqs. (18)-(20), we get

$$
\begin{aligned}
w(t)= & \vartheta_{2}(0) e^{-(b+d) t} \\
& +(1-n) \int_{0}^{t} e^{-(b+d)(t-\theta)}\left(\int_{0}^{\zeta_{1}} f_{1}(\delta) e^{-a_{1} \delta} s(\theta-\delta)\right. \\
& \left.\times\left[\frac{\omega_{1} p(\theta-\delta)}{1+\mu_{1} p(\theta-\delta)}+\frac{\omega_{2} y(\theta-\delta)}{1+\mu_{2} y(\theta-\delta)}\right] d \delta\right) d \theta \geq 0, \\
y(t)= & \vartheta_{3}(0) e^{-\epsilon t} \\
& +n \int_{0}^{t} e^{-\epsilon(t-\theta)}\left(\int_{0}^{\zeta_{2}} f_{2}(\delta) e^{-a_{2} \delta} \delta(\theta-\delta)\right. \\
& \left.\times\left[\frac{\omega_{1} p(\theta-\delta)}{1+\mu_{1} p(\theta-\delta)}+\frac{\omega_{2} y(\theta-\delta)}{1+\mu_{2} y(\theta-\delta)}\right] d \delta+d w(\theta)\right) d \theta \geq 0, \\
p(t)= & \vartheta_{4}(0) e^{-\int_{0}^{t}(c+r x(\varphi)) d \varphi}+\int_{0}^{t} e^{-\int_{\theta}^{t}(c+r x(\varphi)) d \varphi} \int_{0}^{\zeta_{3}} f_{3}(\delta) e^{-a_{3} \delta} \varkappa y(\theta-\delta) d \delta d \theta \\
\geq & 0 \quad \text { for } t \in[0, k] .
\end{aligned}
$$

By recursive argument, we show that $w(t) \geq 0, y(t) \geq 0$, and $p(t) \geq 0$ for all $t \geq 0$. For the boundedness of the solution, we have from Eq. (17) $\lim _{t \rightarrow \infty} \sup s(t) \leq \frac{\varrho}{\xi}$. Let us consider

$$
g_{1}(t)=(1-n) \int_{0}^{\zeta_{1}} f_{1}(\delta) e^{-a_{1} \delta} s(t-\delta) d \delta+n \int_{0}^{\zeta_{2}} f_{2}(\delta) e^{-a_{2} \delta} s(t-\delta) d \delta+w(t)+y(t)
$$

then

$$
\begin{aligned}
\dot{g}_{1}(t)= & (1-n) \int_{0}^{\zeta_{1}} f_{1}(\delta) e^{-a_{1} \delta}[\varrho-\xi s(t-\delta)-s(t-\delta) \\
& \left.\times\left(\frac{\omega_{1} p(t-\delta)}{1+\mu_{1} p(t-\delta)}+\frac{\omega_{2} y(t-\delta)}{1+\mu_{2} y(t-\delta)}\right)\right] d \delta \\
& +n \int_{0}^{\zeta_{2}} f_{2}(\delta) e^{-a_{2} \delta}[\varrho-\xi s(t-\delta)-s(t-\delta) \\
& \left.\times\left(\frac{\omega_{1} p(t-\delta)}{1+\mu_{1} p(t-\delta)}+\frac{\omega_{2} y(t-\delta)}{1+\mu_{2} y(t-\delta)}\right)\right] d \delta \\
& +(1-n) \int_{0}^{\zeta_{1}} f_{1}(\delta) e^{-a_{1} \delta} s(t-\delta)\left(\frac{\omega_{1} p(t-\delta)}{1+\mu_{1} p(t-\delta)}+\frac{\omega_{2} y(t-\delta)}{1+\mu_{2} y(t-\delta)}\right) d \delta \\
& -(b+d) w(t) \\
& +n \int_{0}^{\zeta_{2}} f_{2}(\delta) e^{-a_{2} \delta} s(t-\delta)\left(\frac{\omega_{1} p(t-\delta)}{1+\mu_{1} p(t-\delta)}+\frac{\omega_{2} y(t-\delta)}{1+\mu_{2} y(t-\delta)}\right) d \delta+d w(t)-\epsilon y(t) \\
= & \varrho(1-n) \int_{0}^{\zeta_{1}} f_{1}(\delta) e^{-a_{1} \delta} d \delta+\varrho n \int_{0}^{\zeta_{2}} f_{2}(\delta) e^{-a_{2} \delta} d \delta \\
& -\xi(1-n) \int_{0}^{\zeta_{1}} f_{1}(\delta) e^{-a_{1} \delta} s(t-\delta) d \delta-\xi n \int_{0}^{\zeta_{2}} f_{2}(\delta) e^{-a_{2} \delta} s(t-\delta) d \delta \\
& -b w(t)-\epsilon y(t)
\end{aligned}
$$




$$
\begin{aligned}
& \leq \varrho-\sigma_{1}\left((1-n) \int_{0}^{\zeta_{1}} f_{1}(\delta) e^{-a_{1} \delta} s(t-\delta) d \delta+\int_{0}^{\zeta_{2}} f_{2}(\delta) e^{-a_{2} \delta} s(t-\delta) d \delta+w(t)+y(t)\right) \\
& =\varrho-\sigma_{1} g_{1}(t) .
\end{aligned}
$$

It follows that $\lim _{t \rightarrow \infty} \sup g_{1}(t) \leq \Delta_{1}$. Since $s(t)>0, w(t) \geq 0$, and $y(t) \geq 0$, then $\lim _{t \rightarrow \infty} \sup w(t) \leq \Delta_{1}$ and $\lim _{t \rightarrow \infty} \sup y(t) \leq \Delta_{1}$. Moreover, we define

$$
g_{2}(t)=p(t)+\frac{r}{\rho} x(t)
$$

then

$$
\begin{aligned}
\dot{g}_{2}(t) & =\varkappa \int_{0}^{\zeta_{3}} f_{3}(\delta) e^{-a_{3} \delta} y(t-\delta) d \delta-c p+\frac{r}{\rho} \lambda-\frac{r}{\rho} m x \\
& \leq \varkappa \Delta_{1}+\frac{r}{\rho} \lambda-\sigma_{2}\left(p(t)+\frac{r}{\rho} x(t)\right) \\
& =\frac{\varkappa \rho \Delta_{1}+r \lambda}{\rho}-\sigma_{2} g_{2}(t) .
\end{aligned}
$$

Then $\lim _{t \rightarrow \infty} \sup g_{2}(t) \leq \Delta_{2}$. The nonnegativity of the solution implies that $\lim _{t \rightarrow \infty} \sup p(t) \leq \Delta_{2}$ and $\lim _{t \rightarrow \infty} \sup x(t) \leq \Delta_{3}$. This shows the ultimate boundedness of $s(t), w(t), y(t), p(t)$, and $x(t)$.

\subsection{Equilibria}

Define the basic reproduction number

$$
\mathcal{R}_{0}^{D}=\frac{\alpha_{1}^{D}\left(m \varkappa \omega_{1} F_{3}+c m \omega_{2}+r \lambda \omega_{2}\right) \varrho}{\xi \epsilon(c m+r \lambda)},
$$

where $\alpha_{1}^{D}=\frac{d}{b+d}(1-n) F_{1}+n F_{2}$.

Lemma 4 If $\mathcal{R}_{0}^{D} \leq 1$, then system (17)-(21) has only one equilibrium $\Omega_{0}$, and if $\mathcal{R}_{0}^{D}>1$, then the system has two equilibria $\Omega_{0}$ and $\Omega_{1}$.

The proof of Lemma 4 is given in the Appendix.

\subsection{Global properties}

Theorem 3 For system (17)-(21), if $\mathcal{R}_{0}^{D} \leq 1$, then $\Omega_{0}$ is G.A.S.

Proof Let a function $V_{0}(s, w, y, p, x)$ be defined as follows:

$$
\begin{aligned}
V_{0}= & \alpha_{1}^{D} s_{0} G\left(\frac{s}{s_{0}}\right)+\frac{d}{b+d} w+y+\frac{\alpha_{1}^{D} \omega_{1} s_{0}}{c+r x_{0}} p+\frac{r \alpha_{1}^{D} \omega_{1} s_{0}}{\rho\left(c+r x_{0}\right)} x_{0} G\left(\frac{x}{x_{0}}\right) \\
& +\frac{d(1-n)}{b+d} \int_{0}^{\zeta_{1}} f_{1}(\delta) e^{-a_{1} \delta} \int_{0}^{\delta}\left[\frac{\omega_{1} s(t-\theta) p(t-\theta)}{1+\mu_{1} p(t-\theta)}+\frac{\omega_{2} s(t-\theta) y(t-\theta)}{1+\mu_{2} y(t-\theta)}\right] d \theta d \delta \\
& +n \int_{0}^{\zeta_{2}} f_{2}(\delta) e^{-a_{2} \delta} \int_{0}^{\delta}\left[\frac{\omega_{1} s(t-\theta) p(t-\theta)}{1+\mu_{1} p(t-\theta)}+\frac{\omega_{2} s(t-\theta) y(t-\theta)}{1+\mu_{2} y(t-\theta)}\right] d \theta d \delta \\
& +\frac{\alpha_{1}^{D} \omega_{1} s_{0} \varkappa}{c+r x_{0}} \int_{0}^{\zeta_{3}} f_{3}(\delta) e^{-a_{3} \delta} \int_{0}^{\delta} y(t-\theta) d \theta d \delta .
\end{aligned}
$$


Calculating $\frac{d V_{0}}{d t}$ along system (17)-(21), we obtain

$$
\begin{aligned}
\frac{d V_{0}}{d t}= & \alpha_{1}^{D}\left(1-\frac{s_{0}}{s}\right)\left(\varrho-\xi s-\frac{\omega_{1} s p}{1+\mu_{1} p}-\frac{\omega_{2} s y}{1+\mu_{2} y}\right) \\
& +\frac{d}{b+d}\left[(1-n) \int_{0}^{\zeta_{1}} f_{1}(\delta) e^{-a_{1} \delta}\left[\frac{\omega_{1} s(t-\delta) p(t-\delta)}{1+\mu_{1} p(t-\delta)}+\frac{\omega_{2} s(t-\delta) y(t-\delta)}{1+\mu_{2} y(t-\delta)}\right] d \delta\right. \\
& -(b+d) w] \\
& +n \int_{0}^{\zeta_{2}} f_{2}(\delta) e^{-a_{2} \delta}\left[\frac{\omega_{1} s(t-\delta) p(t-\delta)}{1+\mu_{1} p(t-\delta)}+\frac{\omega_{2} s(t-\delta) y(t-\delta)}{1+\mu_{2} y(t-\delta)}\right] d \delta+d w-\epsilon y \\
& +\frac{\alpha_{1}^{D} \omega_{1} s_{0}}{c+r x_{0}}\left(\varkappa \int_{0}^{\zeta_{3}} f_{3}(\delta) e^{-a_{3} \delta} y(t-\delta) d \delta-c p-r x p\right) \\
& +\frac{r \alpha_{1}^{D} \omega_{1} s_{0}}{\rho\left(c+r x_{0}\right)}\left(1-\frac{x_{0}}{x}\right)\left(\lambda+\rho x p-m_{x}\right) \\
& +\frac{d(1-n)}{b+d} \int_{0}^{\zeta_{1}} f_{1}(\delta) e^{-a_{1} \delta}\left[\frac{\omega_{1} s p}{1+\mu_{1} p}+\frac{\omega_{2} s y}{1+\mu_{2} y}-\frac{\omega_{1} s(t-\delta) p(t-\delta)}{1+\mu_{1} p(t-\delta)}\right. \\
& +\frac{\alpha_{1}^{D} \omega_{1} s_{0}}{c+r x_{0}} \varkappa \int_{0}^{\zeta_{3}} f_{3}(\delta) e^{-a_{3} \delta}(y-y(t-\delta)) d \delta . \\
& +n \int_{0}^{\zeta_{2}} f_{2}(\delta) e^{-a_{2} \delta}\left[\frac{\omega_{1} s p}{1+\mu_{2} y(t-\delta)}+\frac{\omega_{2} s y}{1+\mu_{2} y}-\frac{\omega_{1} s(t-\delta) p(t-\delta)}{1+\mu_{1} p(t-\delta)}\right. \\
& \left.\frac{\omega_{2} s(t-\delta) y(t-\delta)}{1+\mu_{2} y(t-\delta)}\right] d \delta
\end{aligned}
$$

Collecting terms of Eq. (22), we get

$$
\begin{aligned}
\frac{d V_{0}}{d t}= & -\alpha_{1}^{D} \xi \frac{\left(s-s_{0}\right)^{2}}{s}-\frac{\alpha_{1}^{D} \omega_{1} s_{0} \mu_{1} p^{2}}{1+\mu_{1} p}-\frac{\alpha_{1}^{D} \omega_{2} s_{0} \mu_{2} y^{2}}{1+\mu_{2} y}+\epsilon\left(\frac{\alpha_{1}^{D} \omega_{2} s_{0}}{\epsilon}+\frac{\alpha_{1}^{D} \omega_{1} s_{0} \varkappa F_{3}}{\epsilon\left(c+r x_{0}\right)}-1\right) y \\
& +\frac{r \alpha_{1}^{D} \omega_{1} s_{0}}{\rho\left(c+r x_{0}\right)}\left(1-\frac{x_{0}}{x}\right)\left(m x_{0}-m x\right) \\
= & -\alpha_{1}^{D \xi} \frac{\left(s-s_{0}\right)^{2}}{s}-\frac{\alpha_{1}^{D} \omega_{1} s_{0} \mu_{1} p^{2}}{1+\mu_{1} p}-\frac{\alpha_{1}^{D} \omega_{2} s_{0} \mu_{2} y^{2}}{1+\mu_{2} y}-\frac{r \alpha_{1}^{D} \omega_{1} s_{0} m}{\rho\left(c+r x_{0}\right)} \frac{\left(x-x_{0}\right)^{2}}{x} \\
& +\epsilon\left(\mathcal{R}_{0}^{D}-1\right) y .
\end{aligned}
$$

If $\mathcal{R}_{0}^{D} \leq 1$, then $\frac{d V_{0}}{d t} \leq 0$ for all $s, w, y, p, x>0$. Moreover, $\frac{d V_{0}}{d t}=0$ when $s=s_{0}, x=x_{0}$, and $y=p=0$. Similar to the proof of Theorem 1 , one can show that $\frac{d V_{0}}{d t}=0$ at $\Omega_{0}$. Applying LaSalle's invariance principle [58], we get that if $R_{0}^{D} \leq 1$, then $\Omega_{0}$ is G.A.S.

Theorem 4 For system (17)-(21), if $\mathcal{R}_{0}^{D}>1$, then $\Omega_{1}$ is G.A.S.

Proof Let $V_{1}(s, w, y, p, x)$ be defined as follows:

$$
V_{1}=\alpha_{1}^{D} s_{1} G\left(\frac{s}{s_{1}}\right)+\frac{d}{b+d} w_{1} G\left(\frac{w}{w_{1}}\right)+y_{1} G\left(\frac{y}{y_{1}}\right)
$$




$$
\begin{aligned}
& +\frac{\alpha_{1}^{D} \omega_{1} s_{1} p_{1}}{\varkappa F_{3}\left(1+\mu_{1} p_{1}\right) y_{1}} p_{1} G\left(\frac{p}{p_{1}}\right)+\frac{r \alpha_{1}^{D} \omega_{1} s_{1} p_{1}}{\rho \varkappa F_{3}\left(1+\mu_{1} p_{1}\right) y_{1}} x_{1} G\left(\frac{x}{x_{1}}\right) \\
& +\frac{d(1-n)}{b+d} \frac{\omega_{1} s_{1} p_{1}}{1+\mu_{1} p_{1}} \int_{0}^{\zeta_{1}} f_{1}(\delta) e^{-a_{1} \delta} \int_{0}^{\delta} G\left(\frac{s(t-\theta) p(t-\theta)\left(1+\mu_{1} p_{1}\right)}{s_{1} p_{1}\left(1+\mu_{1} p(t-\theta)\right)}\right) d \theta d \delta \\
& +\frac{d(1-n)}{b+d} \frac{\omega_{2} s_{1} y_{1}}{1+\mu_{1} y_{1}} \int_{0}^{\zeta_{1}} f_{1}(\delta) e^{-a_{1} \delta} \int_{0}^{\delta} G\left(\frac{s(t-\theta) y(t-\theta)\left(1+\mu_{2} y_{1}\right)}{s_{1} y_{1}\left(1+\mu_{2} y(t-\theta)\right.}\right) d \theta d \delta \\
& +\frac{n \omega_{1} s_{1} p_{1}}{1+\mu_{1} p_{1}} \int_{0}^{\zeta_{2}} f_{2}(\delta) e^{-a_{2} \delta} \int_{0}^{\delta} G\left(\frac{s(t-\theta) p(t-\theta)\left(1+\mu_{1} p_{1}\right)}{s_{1} p_{1}\left(1+\mu_{1} p(t-\theta)\right)}\right) d \theta d \delta \\
& +\frac{n \omega_{2} s_{1} y_{1}}{1+\mu_{2} y_{1}} \int_{0}^{\zeta_{2}} f_{2}(\delta) e^{-a_{2} \delta} \int_{0}^{\delta} G\left(\frac{s(t-\theta) y(t-\theta)\left(1+\mu_{2} y_{1}\right)}{s_{1} y_{1}\left(1+\mu_{2} y(t-\theta)\right.}\right) d \theta d \delta \\
& +\frac{\alpha_{1}^{D} \omega_{1} s_{1} p_{1}}{F_{3}\left(1+\mu_{1} p_{1}\right)} \int_{0}^{\zeta_{3}} f_{3}(\delta) e^{-a_{3} \delta} \int_{0}^{\delta} G\left(\frac{y(t-\theta)}{y_{1}}\right) d \theta d \delta .
\end{aligned}
$$

Then

$$
\begin{aligned}
& \frac{d V_{1}}{d t}=\alpha_{1}^{D}\left(1-\frac{s_{1}}{s}\right)\left(\varrho-\xi s-\frac{\omega_{1} s p}{1+\mu_{1} p_{1}}-\frac{\omega_{2} s y}{1+\mu_{2} y_{1}}\right) \\
& +\frac{d}{b+d}\left(1-\frac{w_{1}}{w}\right)\left[( 1 - n ) \int _ { 0 } ^ { \zeta _ { 1 } } f _ { 1 } ( \delta ) e ^ { - a _ { 1 } \delta } \left(\frac{\omega_{1} s(t-\delta) p(t-\delta)}{1+\mu_{1} p(t-\delta)}\right.\right. \\
& \left.\left.+\frac{\omega_{2} s(t-\delta) y(t-\delta)}{1+\mu_{2} y(t-\delta)}\right) d \delta-(b+d) w\right] \\
& +\left(1-\frac{y_{1}}{y}\right)\left[n \int _ { 0 } ^ { \zeta _ { 2 } } f _ { 2 } ( \delta ) e ^ { - a _ { 2 } \delta } \left(\frac{\omega_{1} s(t-\delta) p(t-\delta)}{1+\mu_{1} p(t-\delta)}\right.\right. \\
& \left.\left.+\frac{\omega_{2} s(t-\delta) y(t-\delta)}{1+\mu_{2} y(t-\delta)}\right) d \delta+d w-\epsilon y\right] \\
& +\frac{\alpha_{1}^{D} \omega_{1} s_{1} p_{1}}{\varkappa F_{3}\left(1+\mu_{1} p_{1}\right) y_{1}}\left(1-\frac{p_{1}}{p}\right)\left[\varkappa \int_{0}^{\zeta_{3}} f_{3}(\delta) e^{-a_{3} \delta} y(t-\delta) d \delta-c p-r x p\right] \\
& +\frac{r \alpha_{1}^{D} \omega_{1} s_{1} p_{1}}{\rho \varkappa F_{3}\left(1+\mu_{1} p_{1}\right) y_{1}}\left(1-\frac{x_{1}}{x}\right)(\lambda+\rho x p-m x) \\
& +\frac{d(1-n)}{b+d} \int_{0}^{\zeta_{1}} f_{1}(\delta) e^{-a_{1} \delta}\left[\frac{\omega_{1} s p}{1+\mu_{1} p}-\frac{\omega_{1} s(t-\delta) p(t-\delta)}{\left(1+\mu_{1} p(t-\delta)\right)}\right. \\
& \left.+\frac{\omega_{1} s_{1} p_{1}}{1+\mu_{1} p_{1}} \ln \left(\frac{s(t-\delta) p(t-\delta)\left(1+\mu_{1} p\right)}{s p\left(1+\mu_{1} p(t-\delta)\right)}\right)\right] d \delta \\
& +\frac{d(1-n)}{b+d} \int_{0}^{\zeta_{1}} f_{1}(\delta) e^{-a_{1} \delta}\left[\frac{\omega_{2} s y}{1+\mu_{2} y}-\frac{\omega_{2} s(t-\delta) y(t-\delta)}{\left(1+\mu_{2} y(t-\delta)\right)}\right. \\
& \left.+\frac{\omega_{2} s_{1} y_{1}}{1+\mu_{2} y_{1}} \ln \left(\frac{s(t-\delta) y(t-\delta)\left(1+\mu_{2} y\right)}{s y\left(1+\mu_{2} y(t-\delta)\right)}\right)\right] d \delta \\
& +n \int_{0}^{\zeta_{2}} f_{2}(\delta) e^{-a_{2} \delta}\left[\frac{\omega_{1} s p}{1+\mu_{1} p}-\frac{\omega_{1} s(t-\delta) p(t-\delta)}{\left(1+\mu_{1} p(t-\delta)\right)}\right. \\
& \left.+\frac{\omega_{1} s_{1} p_{1}}{1+\mu_{1} p_{1}} \ln \left(\frac{s(t-\delta) p(t-\delta)\left(1+\mu_{1} p\right)}{s p\left(1+\mu_{1} p(t-\delta)\right)}\right)\right] d \delta \\
& +n \int_{0}^{\zeta_{2}} f_{2}(\delta) e^{-a_{2} \delta}\left[\frac{\omega_{2} s y}{1+\mu_{2} y}-\frac{\omega_{2} s(t-\delta) y(t-\delta)}{\left(1+\mu_{2} y(t-\delta)\right)}\right.
\end{aligned}
$$




$$
\begin{aligned}
& \left.+\frac{\omega_{2} s_{1} y_{1}}{1+\mu_{2} y_{1}} \ln \left(\frac{s(t-\delta) y(t-\delta)\left(1+\mu_{2} y\right)}{s y\left(1+\mu_{2} y(t-\delta)\right)}\right)\right] d \delta \\
& +\frac{\alpha_{1}^{D} \omega_{1} s_{1} p_{1}}{F_{3}\left(1+\mu_{1} p_{1}\right)} \int_{0}^{\zeta_{3}} f_{3}(\delta) e^{-a_{3} \delta}\left[\frac{y}{y_{1}}-\frac{y(t-\delta)}{y_{1}}+\ln \left(\frac{y(t-\delta)}{y_{1}}\right)\right] d \delta .
\end{aligned}
$$

Simplify Eq. (24) as follows:

$$
\begin{aligned}
& \frac{d U_{1}}{d t}=\alpha_{1}^{D}\left(1-\frac{s_{1}}{s}\right)(\varrho-\xi s)+\alpha_{1}^{D}\left(\frac{\omega_{1} s_{1} p}{1+\mu_{1} p}+\frac{\omega_{2} s_{1} y}{1+\mu_{2} y}\right) \\
& -\frac{d(1-n)}{b+d} \int_{0}^{\zeta_{1}} f_{1}(\delta) e^{-a_{1} \delta_{1}} \\
& \times\left[\frac{\omega_{1} s(t-\delta) p(t-\delta) w_{1}}{1+\mu_{1} p(t-\delta) w}+\frac{\omega_{2} s(t-\delta) y(t-\delta) w_{1}}{1+\mu_{2} y(t-\delta) w}\right] d \delta+d w_{1} \\
& -n \int_{0}^{\zeta_{2}} f_{2}(\delta) e^{-a_{2} \delta}\left[\frac{\omega_{1} s(t-\delta) p(t-\delta) y_{1}}{\left(1+\mu_{1} p(t-\delta)\right) y}+\frac{\omega_{2} s(t-\delta) y(t-\delta) y_{1}}{\left(1+\mu_{2} y(t-\delta)\right) y}\right] d \delta-d w \frac{y_{1}}{y} \\
& -\epsilon y+\epsilon y_{1} \\
& -\frac{\alpha_{1}^{D} \omega_{1} s_{1} p_{1}}{F_{3}\left(1+\mu_{1} p_{1}\right)} \int_{0}^{\zeta_{3}} f_{3}(\delta) e^{-a_{3} \delta}\left(\frac{y(t-\delta) p_{1}}{y_{1} p}\right) d \delta-\frac{\alpha_{1}^{D} \omega_{1} s_{1} p_{1}}{\varkappa F_{3}\left(1+\mu_{1} p_{1}\right) y_{1}} c p \\
& +\frac{\alpha_{1}^{D} \omega_{1} s_{1} p_{1}}{\varkappa F_{3}\left(1+\mu_{1} p_{1}\right) y_{1}} c p_{1} \\
& +\frac{\alpha_{1}^{D} \omega_{1} s_{1} p_{1}}{\varkappa F_{3}\left(1+\mu_{1} p_{1}\right) y_{1}} r x p_{1}-\frac{\alpha_{1}^{D} \omega_{1} s_{1} p_{1}}{\varkappa F_{3}\left(1+\mu_{1} p_{1}\right) y_{1}} r x_{1} p \\
& +\frac{r \alpha_{1}^{D} \omega_{1} s_{1} p_{1}}{\rho \varkappa F_{3}\left(1+\mu_{1} p_{1}\right) y_{1}}\left(1-\frac{x_{1}}{x}\right)(\lambda-m x) \\
& +\frac{d(1-n)}{b+d} \int_{0}^{\zeta_{1}} f_{1}(\delta) e^{-a_{1} \delta}\left[\frac{\omega_{1} s_{1} p_{1}}{1+\mu_{1} p_{1}} \ln \left(\frac{s(t-\delta) p(t-\delta)\left(1+\mu_{1} p\right)}{s p\left(1+\mu_{1} p(t-\delta)\right)}\right)\right. \\
& \left.+\frac{\omega_{2} s_{1} y_{1}}{1+\mu_{2} y_{1}} \ln \left(\frac{s(t-\delta) y(t-\delta)\left(1+\mu_{2} y\right)}{s y\left(1+\mu_{2} y(t-\delta)\right)}\right)\right] d \delta \\
& +n \int_{0}^{\zeta_{2}} f_{2}(\delta) e^{-a_{2} \delta}\left[\frac{\omega_{1} s_{1} p_{1}}{1+\mu_{1} p_{1}} \ln \left(\frac{s(t-\delta) p(t-\delta)\left(1+\mu_{1} p\right)}{s p\left(1+\mu_{1} p(t-\delta)\right)}\right)\right. \\
& \left.+\frac{\omega_{2} s_{1} y_{1}}{1+\mu_{2} y_{1}} \ln \left(\frac{s(t-\delta) y(t-\delta)\left(1+\mu_{2} y\right)}{s y\left(1+\mu_{2} y(t-\delta)\right)}\right)\right] d \delta \\
& +\frac{\alpha_{1}^{D} \omega_{1} s_{1} p_{1}}{F_{3}\left(1+\mu_{1} p_{1}\right)} \int_{0}^{\zeta_{3}} f_{3}(\delta) e^{-a_{3} \delta}\left[\frac{y}{y_{1}}+\ln \left(\frac{y(t-\delta)}{y}\right)\right] d \delta .
\end{aligned}
$$

Applying the equilibrium conditions for $\Omega_{1}$ :

$$
\begin{aligned}
& \varrho=\xi s_{1}+\frac{\omega_{1} s_{1} p_{1}}{1+\mu_{1} p_{1}}+\frac{\omega_{2} s_{1} y_{1}}{1+\mu_{2} y_{1}}, \quad w_{1}=\frac{(1-n) F_{1}}{(b+d)}\left(\frac{\omega_{1} s_{1} p_{1}}{1+\mu_{1} p_{1}}+\frac{\omega_{2} s_{1} y_{1}}{1+\mu_{2} y_{1}}\right), \\
& \epsilon y_{1}=\frac{d(1-n) F_{1}}{b+d}\left(\frac{\omega_{1} s_{1} p_{1}}{1+\mu_{1} p_{1}}+\frac{\omega_{2} s_{1} y_{1}}{1+\mu_{2} y_{1}}\right)+n F_{2}\left(\frac{\omega_{1} s_{1} p_{1}}{1+\mu_{1} p_{1}}+\frac{\omega_{2} s_{1} y_{1}}{1+\mu_{2} y_{1}}\right) \\
& =\alpha_{1}^{D}\left(\frac{\omega_{1} s_{1} p_{1}}{1+\mu_{1} p_{1}}+\frac{\omega_{2} s_{1} y_{1}}{1+\mu_{2} y_{1}}\right), \\
& c p_{1}=\varkappa F_{3} y_{1}-r x_{1} p_{1}, \quad \lambda=m x_{1}-\rho x_{1} p_{1},
\end{aligned}
$$


we get

$$
\begin{aligned}
& \frac{d U_{1}}{d t}=\alpha_{1}^{D}\left(1-\frac{s_{1}}{s}\right)\left(\xi s_{1}-\xi s\right)+\frac{d(1-n) F_{1}}{b+d} \frac{\omega_{1} s_{1} p_{1}}{1+\mu_{1} p_{1}}\left(1-\frac{s_{1}}{s}\right)+n F_{2} \frac{\omega_{1} s_{1} p_{1}}{1+\mu_{1} p_{1}}\left(1-\frac{s_{1}}{s}\right) \\
& +\frac{d(1-n) F_{1}}{b+d} \frac{\omega_{2} s_{1} y_{1}}{1+\mu_{2} y_{1}}\left(1-\frac{s_{1}}{s}\right)+n F_{2} \frac{\omega_{2} s_{1} y_{1}}{1+\mu_{2} y_{1}}\left(1-\frac{s_{1}}{s}\right) \\
& +\frac{\alpha_{1}^{D} \omega_{1} s_{1} p_{1}}{1+\mu_{1} p_{1}} \frac{\left(1+\mu_{1} p_{1}\right) p}{\left(1+\mu_{1} p\right) p_{1}}+\frac{\alpha_{1}^{D} \omega_{2} s_{1} y_{1}}{1+\mu_{2} y_{1}} \frac{\left(1+\mu_{2} y_{1}\right) y}{\left(1+\mu_{2} y\right) y_{1}} \\
& -\frac{d(1-n)}{b+d} \int_{0}^{\zeta_{1}} f_{1}(\delta) e^{-a_{1} \delta} \frac{\omega_{1} s_{1} p_{1}}{1+\mu_{1} p_{1}}\left[\frac{s(t-\delta) p(t-\delta)\left(1+\mu_{1} p_{1}\right) w_{1}}{s_{1} p_{1}\left(1+\mu_{1} p(t-\delta)\right) w}\right] d \delta \\
& -\frac{d(1-n)}{b+d} \int_{0}^{\zeta_{1}} f_{1}(\delta) e^{-a_{1} \delta} \frac{\omega_{2} s_{1} y_{1}}{1+\mu_{2} y_{1}}\left[\frac{s(t-\delta) y(t-\delta)\left(1+\mu_{2} y_{1}\right) w_{1}}{s_{1} y_{1}\left(1+\mu_{2} y(t-\delta)\right) w}\right] d \delta \\
& +\frac{d(1-n)}{b+d} F_{1}\left[\frac{\omega_{1} s_{1} p_{1}}{1+\mu_{1} p_{1}}+\frac{\omega_{2} s_{1} y_{1}}{1+\mu_{2} y_{1}}\right] \\
& -n \int_{0}^{\zeta_{2}} f_{2}(\delta) e^{-a_{2} \delta} \frac{\omega_{1} s_{1} p_{1}}{1+\mu_{1} p_{1}}\left[\frac{s(t-\delta) p(t-\delta)\left(1+\mu_{1} p_{1}\right) y_{1}}{s_{1} p_{1}\left(1+\mu_{1} p(t-\delta)\right) y}\right] d \delta \\
& -n \int_{0}^{\zeta_{2}} f_{2}(\delta) e^{-a_{2} \delta} \frac{\omega_{2} s_{1} y_{1}}{1+\mu_{2} y_{1}}\left[\frac{s(t-\delta) y(t-\delta)\left(1+\mu_{2} y_{1}\right) y_{1}}{s_{1} y_{1}\left(1+\mu_{2} y(t-\delta)\right) y}\right] d \delta \\
& -\frac{d(1-n)}{b+d} F_{1}\left[\frac{\omega_{1} s_{1} p_{1}}{1+\mu_{1} p_{1}}+\frac{\omega_{2} s_{1} y_{1}}{1+\mu_{2} y_{1}}\right] \frac{w y_{1}}{w_{1} y}-\frac{\alpha_{1}^{D} \omega_{2} s_{1} y_{1}}{1+\mu_{2} y_{1}} \frac{y}{y_{1}} \\
& +\frac{d(1-n)}{b+d} F_{1}\left[\frac{\omega_{1} s_{1} p_{1}}{1+\mu_{1} p_{1}}+\frac{\omega_{2} s_{1} y_{1}}{1+\mu_{2} y_{1}}\right] \\
& +n F_{2}\left[\frac{\omega_{1} s_{1} p_{1}}{1+\mu_{1} p_{1}}+\frac{\omega_{2} s_{1} y_{1}}{1+\mu_{2} y_{1}}\right]-\frac{\alpha_{1}^{D} \omega_{1} s_{1} p_{1}}{F_{3}\left(1+\mu_{1} p_{1}\right)} \int_{0}^{\zeta_{3}} f_{3}(\delta) e^{-a_{3} \delta}\left[\frac{y(t-\delta) p_{1}}{y_{1} p}\right] d \delta \\
& -\frac{\alpha_{1}^{D} \omega_{1} s_{1} p_{1}}{1+\mu_{1} p_{1}} \frac{p}{p_{1}} \\
& +\frac{d(1-n)}{b+d} F_{1} \frac{\omega_{1} s_{1} p_{1}}{1+\mu_{1} p_{1}}+n F_{2} \frac{\omega_{1} s_{1} p_{1}}{1+\mu_{1} p_{1}}-\frac{2 r \alpha_{1}^{D} s_{1} p_{1} \omega_{1}}{\varkappa F_{3}\left(1+\mu_{1} p_{1}\right) y_{1}} x_{1} p_{1} \\
& +\frac{r \alpha_{1}^{D} s_{1} p_{1} \omega_{1}}{\varkappa F_{3}\left(1+\mu_{1} p_{1}\right) y_{1}} x_{1} p_{1}\left(\frac{x}{x_{1}}\right)+\frac{r \alpha_{1}^{D} s_{1} p_{1} \omega_{1}}{\varkappa F_{3}\left(1+\mu_{1} p_{1}\right) y_{1}} x_{1} p_{1}\left(\frac{x_{1}}{x}\right) \\
& -\frac{r \alpha_{1}^{D} s_{1} p_{1} m \omega_{1}}{\rho \varkappa F_{3}\left(1+\mu_{1} p_{1}\right) y_{1}} \frac{\left(x-x_{1}\right)^{2}}{x} \\
& +\frac{d(1-n)}{b+d} \frac{\omega_{1} s_{1} p_{1}}{1+\mu_{1} p_{1}} \int_{0}^{\zeta_{1}} f_{1}(\delta) e^{-a_{1} \delta} \ln \left[\frac{s(t-\delta) p(t-\delta)\left(1+\mu_{1} p\right)}{s p\left(1+\mu_{1} p(t-\delta)\right)}\right] d \delta \\
& +\frac{d(1-n)}{b+d} \frac{\omega_{2} s_{1} y_{1}}{1+\mu_{2} y_{1}} \int_{0}^{\zeta_{1}} f_{1}(\delta) e^{-a_{1} \delta} \ln \left[\frac{s(t-\delta) y(t-\delta)\left(1+\mu_{2} y\right)}{s y\left(1+\mu_{2} y(t-\delta)\right)}\right] d \delta \\
& +\frac{n \omega_{1} s_{1} p_{1}}{1+\mu_{1} p_{1}} \int_{0}^{\zeta_{2}} f_{2}(\delta) e^{-a_{2} \delta} \ln \left[\frac{s(t-\delta) p(t-\delta)\left(1+\mu_{1} p\right)}{s p\left(1+\mu_{1} p(t-\delta)\right)}\right] d \delta \\
& +\frac{n \omega_{2} s_{1} y_{1}}{1+\mu_{2} y_{1}} \int_{0}^{\zeta_{2}} f_{2}(\delta) e^{-a_{2} \delta} \ln \left[\frac{s(t-\delta) y(t-\delta)\left(1+\mu_{2} y\right)}{s y\left(1+\mu_{2} y(t-\delta)\right)}\right] d \delta \\
& +\frac{\alpha_{1}^{D} \omega_{1} s_{1} p_{1}}{F_{3}\left(1+\mu_{1} p_{1}\right)} \int_{0}^{\zeta_{3}} f_{3}(\delta) e^{-a_{3} \delta} \ln \left[\frac{y(t-\delta)}{y}\right] d \delta .
\end{aligned}
$$


Using the following equations:

$$
\begin{aligned}
\ln \left(\frac{s(t-\delta) p(t-\delta)\left(1+\mu_{1} p\right)}{s p\left(1+\mu_{1} p(t-\delta)\right)}\right)= & \ln \left(\frac{s_{1}}{s}\right)+\ln \left(\frac{s(t-\delta) p(t-\delta)\left(1+\mu_{1} p_{1}\right) w_{1}}{s_{1} p_{1}\left(1+\mu_{1} p(t-\delta)\right) w}\right) \\
& +\ln \left(\frac{w y_{1}}{w_{1} y}\right)+\ln \left(\frac{y p_{1}}{y_{1} p}\right)+\ln \left(\frac{1+\mu_{1} p}{1+\mu_{1} p_{1}}\right), \\
\ln \left(\frac{s(t-\delta) y(t-\delta)\left(1+\mu_{2} y\right)}{s y\left(1+\mu_{2} y(t-\delta)\right)}\right)= & \ln \left(\frac{s_{1}}{s}\right)+\ln \left(\frac{s(t-\delta) y(t-\delta)\left(1+\mu_{2} y_{1}\right) w_{1}}{s_{1} y_{1}\left(1+\mu_{2} y(t-\delta)\right) w}\right) \\
& +\ln \left(\frac{w y_{1}}{w_{1} y}\right)+\ln \left(\frac{1+\mu_{2} y}{1+\mu_{2} y_{1}}\right), \\
\ln \left(\frac{s(t-\delta) p(t-\delta)\left(1+\mu_{1} p\right)}{s p\left(1+\mu_{1} p(t-\delta)\right)}\right)= & \ln \left(\frac{s_{1}}{s}\right)+\ln \left(\frac{s(t-\delta) p(t-\delta)\left(1+\mu_{1} p_{1}\right) y_{1}}{s_{1} p_{1}\left(1+\mu_{1} p(t-\delta)\right) y}\right) \\
& +\ln \left(\frac{y p_{1}}{y_{1} p}\right)+\ln \left(\frac{1+\mu_{1} p}{1+\mu_{1} p_{1}}\right), \\
\ln \left(\frac{s(t-\delta) y(t-\delta)\left(1+\mu_{2} y\right)}{s y\left(1+\mu_{2} y(t-\delta)\right)}\right)= & \ln \left(\frac{s_{1}}{s}\right)+\ln \left(\frac{s(t-\delta) y(t-\delta)\left(1+\mu_{2} y_{1}\right)}{s_{1} y\left(1+\mu_{2} y(t-\delta)\right)}\right) \\
& +\ln \left(\frac{1+\mu_{2} y}{1+\mu_{2} y_{1}}\right), \\
\ln \left(\frac{y(t-\delta)}{y}\right)=\ln \left(\frac{y(t-\delta) p_{1}}{y_{1} p}\right)+ & \ln \left(\frac{y_{1} p}{y p_{1}}\right),
\end{aligned}
$$

we get

$$
\begin{aligned}
\frac{d U_{1}}{d t}= & -\alpha_{1}^{D} \xi \frac{\left(s-s_{1}\right)^{2}}{s}-\frac{r \alpha_{1}^{D} s_{1} p_{1} m \omega_{1}}{\rho \varkappa F_{3}\left(1+\mu_{1} p_{1}\right) y_{1}} \frac{\left(x-x_{1}\right)^{2}}{x} \\
& -\frac{r \alpha_{1}^{D} s_{1} p_{1} \omega_{1}}{\varkappa F_{3}\left(1+\mu_{1} p_{1}\right) y_{1}} x_{1} p_{1}\left[2-\frac{x}{x_{1}}-\frac{x_{1}}{x}\right] \\
& +\frac{d(1-n)}{b+d} F_{1}\left(\frac{\omega_{1} s_{1} p_{1}}{1+\mu_{1} p_{1}}+\frac{\omega_{2} s_{1} y_{1}}{1+\mu_{2} y_{1}}\right)\left[1-\frac{s_{1}}{s}+\ln \left(\frac{s_{1}}{s}\right)\right] \\
& +n F_{2}\left(\frac{\omega_{1} s_{1} p_{1}}{1+\mu_{1} p_{1}}+\frac{\omega_{2} s_{1} y_{1}}{1+\mu_{2} y_{1}}\right)\left[1-\frac{s_{1}}{s}+\ln \left(\frac{s_{1}}{s}\right)\right] \\
& +\frac{\alpha_{1}^{D} \omega_{1} s_{1} p_{1}}{1+\mu_{1} p_{1}}\left[\frac{\left(1+\mu_{1} p_{1}\right) p}{\left(1+\mu_{1} p\right) p_{1}}-\frac{p}{p_{1}}-1+\frac{1+\mu_{1} p}{1+\mu_{1} p_{1}}\right] \\
& +\frac{\alpha_{1}^{D} \omega_{2} s_{1} y_{1}}{1+\mu_{2} y_{1}}\left[\frac{\left(1+\mu_{2} y_{1}\right) y}{\left(1+\mu_{2} y\right) y_{1}}-\frac{y}{y_{1}}-1+\frac{1+\mu_{2} y}{1+\mu_{2} y_{1}}\right] \\
& +\frac{d(1-n)}{b+d} \frac{\omega_{1} s_{1} p_{1}}{1+\mu_{1} p_{1}} \int_{0}^{\zeta_{1}} f_{1}(\delta) e^{-a_{1} \delta}\left[1-\frac{s(t-\delta) p(t-\delta)\left(1+\mu_{1} p_{1}\right) w_{1}}{s_{1} p_{1}\left(1+\mu_{1} p\left(t-\delta_{1}\right)\right) w}\right. \\
& \left.+\ln \left(\frac{s(t-\delta) p(t-\delta)\left(1+\mu_{1} p_{1}\right) w_{1}}{s_{1} p_{1}\left(1+\mu_{1} p(t-\delta)\right) w}\right)\right] d \delta \\
& +\frac{d(1-n)}{b+d} \frac{\omega_{2} s_{1} y_{1}}{1+\mu_{2} y_{1}} \int_{0}^{\zeta_{1}} f_{1}(\delta) e^{-a_{1} \delta}\left[1-\frac{s(t-\delta) y(t-\delta)\left(1+\mu_{2} y_{1}\right) w_{1}}{s_{1} y_{1}\left(1+\mu_{2} y(t-\delta)\right) w}\right. \\
& \left.+\ln \left(\frac{s(t-\delta) y(t-\delta)\left(1+\mu_{2} y_{1}\right) w_{1}}{s_{1} y_{1}\left(1+\mu_{2} y(t-\delta)\right) w}\right)\right] d \delta
\end{aligned}
$$




$$
\begin{aligned}
& +n \frac{\omega_{1} s_{1} p_{1}}{1+\mu_{1} p_{1}} \int_{0}^{\zeta_{2}} f_{2}(\delta) e^{-a_{2} \delta}\left[1-\frac{s(t-\delta) p(t-\delta)\left(1+\mu_{1} p_{1}\right) y_{1}}{s_{1} p_{1}\left(1+\mu_{1} p(t-\delta)\right) y}\right. \\
& \left.+\ln \left(\frac{s(t-\delta) p(t-\delta)\left(1+\mu_{1} p_{1}\right) y_{1}}{s_{1} p_{1}\left(1+\mu_{1} p(t-\delta)\right) y}\right)\right] d \delta \\
& +n \frac{\omega_{2} s_{1} y_{1}}{1+\mu_{2} y_{1}} \int_{0}^{\zeta_{2}} f_{2}(\delta) e^{-a_{2} \delta}\left[1-\frac{s(t-\delta) y(t-\delta)\left(1+\mu_{2} y_{1}\right) y_{1}}{s_{1} y_{1}\left(1+\mu_{2} y(t-\delta)\right) y}\right. \\
& \left.+\ln \left(\frac{s(t-\delta) y(t-\delta)\left(1+\mu_{2} y_{1}\right) y_{1}}{s_{1} y_{1}\left(1+\mu_{2} y(t-\delta)\right) y}\right)\right] d \delta \\
& +\frac{d(1-n)}{b+d} F_{1} \frac{\omega_{1} s_{1} p_{1}}{1+\mu_{1} p_{1}}\left[1-\frac{w y_{1}}{w_{1} y}+\ln \left(\frac{w y_{1}}{w_{1} y}\right)\right] \\
& +\frac{d(1-n)}{b+d} F_{1} \frac{\omega_{2} s_{1} y_{1}}{1+\mu_{2} y_{1}}\left[1-\frac{w y_{1}}{w_{1} y}+\ln \left(\frac{w y_{1}}{w_{1} y}\right)\right] \\
& +\frac{\alpha_{1}^{D} \omega_{1} s_{1} p_{1}}{F_{3}\left(1+\mu_{1} p_{1}\right)} \int_{0}^{\zeta_{3}} f_{3}(\delta) e^{-a_{3} \delta}\left[1-\frac{y(t-\delta) p_{1}}{y_{1} p}+\ln \left(\frac{y(t-\delta) p_{1}}{y_{1} p}\right)\right] d \delta \\
& +\frac{\alpha_{1}^{D} \omega_{1} s_{1} p_{1}}{1+\mu_{1} p_{1}}\left[1-\frac{1+\mu_{1} p}{1+\mu_{1} p_{1}}+\ln \left(\frac{1+\mu_{1} p}{1+\mu_{1} p_{1}}\right)\right] \\
& +\frac{\alpha_{1}^{D} \omega_{2} s_{1} y_{1}}{1+\mu_{2} y_{1}}\left[1-\frac{1+\mu_{2} y}{1+\mu_{2} y_{1}}+\ln \left(\frac{1+\mu_{2} y}{1+\mu_{2} y_{1}}\right)\right] .
\end{aligned}
$$

Finally we get

$$
\begin{aligned}
\frac{d U_{1}}{d t}= & -\alpha_{1}^{D} \xi \frac{\left(s-s_{1}\right)^{2}}{s}-\frac{\alpha_{1}^{D} r s_{1} p_{1} \omega_{1}}{\rho \varkappa F_{3}\left(1+\mu_{1} p_{1}\right) y_{1}} \frac{\lambda}{x_{1}} \frac{\left(x-x_{1}\right)^{2}}{x} \\
& -\alpha_{1}^{D}\left(\frac{\omega_{1} s_{1} p_{1}}{1+\mu_{1} p_{1}}+\frac{\omega_{2} s_{1} y_{1}}{1+\mu_{2} y_{1}}\right) G\left(\frac{s_{1}}{s}\right) \\
& -\frac{\alpha_{1}^{D} \omega_{1} s_{1} p_{1}}{1+\mu_{1} p_{1}}\left(\frac{\mu_{1}\left(p-p_{1}\right)^{2}}{\left(1+\mu_{1} p\right)\left(1+\mu_{1} p_{1}\right) p_{1}}\right)-\frac{\alpha_{1}^{D} \omega_{2} s_{1} y_{1}}{1+\mu_{2} y_{1}}\left(\frac{\mu_{2}\left(y-y_{1}\right)^{2}}{\left(1+\mu_{2} y\right)\left(1+\mu_{2} y_{1}\right) y_{1}}\right) \\
& -\frac{d(1-n)}{b+d} \frac{\omega_{1} s_{1} p_{1}}{1+\mu_{1} p_{1}} \int_{0}^{\zeta_{1}} f_{1}(\delta) e^{-a_{1} \delta} G\left(\frac{s(t-\delta) p(t-\delta)\left(1+\mu_{1} p_{1}\right) w_{1}}{s_{1} p_{1}\left(1+\mu_{1} p(t-\delta)\right) w}\right) d \delta \\
& -\frac{d(1-n)}{b+d} \frac{\omega_{2} s_{1} y_{1}}{1+\mu_{2} y_{1}} \int_{0}^{\zeta_{1}} f_{1}(\delta) e^{-a_{1} \delta} G\left(\frac{s(t-\delta) y(t-\delta)\left(1+\mu_{2} y_{1}\right) w_{1}}{s_{1} y_{1}\left(1+\mu_{2} y(t-\delta)\right) w}\right) d \delta \\
& -\frac{n \omega_{1} s_{1} p_{1}}{1+\mu_{1} p_{1}} \int_{0}^{\zeta_{2}} f_{2}(\delta) e^{-a_{2} \delta} G\left(\frac{s(t-\delta) p(t-\delta)\left(1+\mu_{1} p_{1}\right) y_{1}}{s_{1} p_{1}\left(1+\mu_{1} p(t-\delta)\right) y}\right) d \delta \\
& -\frac{n \omega_{2} s_{1} y_{1}}{1+\mu_{2} y_{1}} \int_{0}^{\zeta_{2}} f_{2}(\delta) e^{-a_{2} \delta} G\left(\frac{s(t-\delta) y\left(t-\delta\left(1+\mu_{2} y_{1}\right)\right)}{s_{1}\left(1+\mu_{2} y(t-\delta)\right) y}\right) d \delta \\
& -\frac{d(1-n)}{b+d} F_{1}\left[\frac{\omega_{1} s_{1} p_{1}}{1+\mu_{1} p_{1}}+\frac{\omega_{2} s_{1} y_{1}}{1+\mu_{2} y_{1}}\right] G\left(\frac{w y_{1}}{w_{1} y}\right) \\
& -\frac{\alpha_{1}^{D} \omega_{1} s_{1} p_{1}}{F_{3}\left(1+\mu_{1} p_{1}\right)} \int_{0}^{\zeta_{3}} f_{3}(\delta) e^{-a_{3} \delta} G\left(\frac{y(t-\delta) p_{1}}{y_{1} p}\right)-\frac{\alpha_{1}^{D} \omega_{1} s_{1} p_{1}}{1+\mu_{1} p_{1}} G\left(\frac{1+\mu_{1} p}{1+\mu_{1} p_{1}}\right) \\
& -\frac{\alpha_{1}^{D} \omega_{2} s_{1} y_{1}}{1+\mu_{2} y_{1}} G\left(\frac{1+\mu_{2} y}{1+\mu_{2} y_{1}}\right) .
\end{aligned}
$$

Therefore, $\frac{d V_{1}}{d t} \leq 0$ for all $s, w, y, p, x>0$. Let $L=\left\{(s, w, y, p, x): \frac{d V_{1}}{d t}=0\right\}$ and $Q$ be the largest invariant subset of $L$. The trajectory of model (17)-(21) tends to $Q$ [57]. It can be verified 
that $\frac{d V_{1}}{d t}=0$ implies $s=s_{1}, y=y_{1}, p=p_{1}, x=x_{1}$, and

$$
\begin{aligned}
\frac{s(t-\delta) p(t-\delta)\left(1+\mu_{1} p_{1}\right) w_{1}}{s_{1} p_{1}\left(1+\mu_{1} p(t-\delta)\right) w} & =\frac{s(t-\delta) y(t-\delta)\left(1+\mu_{2} y_{1}\right) w_{1}}{s_{1} y_{1}\left(1+\mu_{2} y(t-\delta)\right) w} \\
& =\frac{s(t-\delta) p(t-\delta)\left(1+\mu_{1} p_{1}\right) y_{1}}{s_{1} p_{1}\left(1+\mu_{1} p(t-\delta)\right) y} \\
& =\frac{s(t-\delta) y\left(t-\delta\left(1+\mu_{2} y_{1}\right)\right)}{s_{1}\left(1+\mu_{2} y(t-\delta)\right) y}=1, \quad \delta \in[0, k] .
\end{aligned}
$$

Applying the above relations to Eq. (19), we get $w(t)=w_{1}$, and hence $Q=\left\{\Omega_{1}\right\}$. Applying LaSalle's invariance principle [58], we get $\Omega_{1}$ is G.A.S. when $R_{0}^{D}>1$.

\section{Numerical simulations}

The previous sections described the analytical results and provided a qualitative interpretation of the solutions of the underlying delay differential equation models. In this section, exhaustive numerical exploration has been done to explore the long-term dynamics of the CHIKV model and the various properties of the solutions. We conduct numerical simulations for system (5)-(9) with values of the parameters listed in Table 1. We consider two cases of the effect of $\delta_{1}, \delta_{2}, \delta_{3}$ and $\mu_{1}, \mu_{2}$ as follows.

Case 1 We let $\delta=\delta_{1}=\delta_{2}=\delta_{3}$ and $\omega_{1}=\omega_{2}=0.05$ by using the following initial conditions:

$$
\begin{array}{ll}
\vartheta_{1}(\varphi)=14, & \vartheta_{2}(\varphi)=0.3, \quad \vartheta_{3}(\varphi)=1.0, \\
\vartheta_{4}(\varphi)=1.5, & \vartheta_{5}(\varphi)=2.5, \quad \varphi \in[-k, 0] .
\end{array}
$$

We can see in Fig. 2, for smaller values of $\delta$, e.g., $\delta=0.0,0.5,1.0$, then $\mathcal{R}_{0}>1$, and the trajectory of the system converges to the equilibrium $\Omega_{1}$; whereas when $\delta$ becomes larger, e.g., $\delta=1.5,1.9$, then $\mathcal{R}_{0} \leq 1$, and the system has one equilibrium $\Omega_{0}$. Also, for this case, the concentrations of the uninfected monocytes and antibodies return to their values, while the CHIKV particles are cleared from the body. Let $\delta^{c t}$ be the critical value of the parameter $\delta$ such that

$$
\mathcal{R}_{0}=\frac{\alpha_{1}\left(m \varkappa \omega_{1} e^{-a_{3} \delta^{c t}}+c m \omega_{2}+r \lambda \omega_{2}\right) \varrho}{\xi \epsilon(c m+r \lambda)}=1 .
$$

Using the data given in Table 1 , we obtain $\delta^{c t}=1.278$. The variations of $\mathcal{R}_{0}$ w.r.t. $\delta$ are listed in Table 2. We can observe that as $\delta$ is increased, $\mathcal{R}_{0}$ is decreased. Moreover, we have the following cases:

Table 1 The value of the parameters of model (5)-(9)

\begin{tabular}{ll}
\hline Parameter & Value \\
\hline$\varrho$ & 2 \\
$\omega_{1}, \omega_{2}$ & varied \\
$\varkappa$ & 4 \\
$r$ & 0.5 \\
$m$ & 1 \\
$b$ & 0.5 \\
$n$ & 0.5 \\
$\delta_{1}, \delta_{2}, \delta_{3}$ & varied \\
\hline
\end{tabular}

\begin{tabular}{ll}
\hline Parameter & Value \\
\hline$\xi$ & 0.1 \\
$\epsilon$ & 0.5 \\
$c$ & 0.1 \\
$\lambda$ & 1.4 \\
$\rho$ & 0.2 \\
$a_{1}=a_{2}=a_{3}$ & 1 \\
$d$ & 0.5 \\
$\mu_{1}, \mu_{2}$ & varied \\
\hline
\end{tabular}




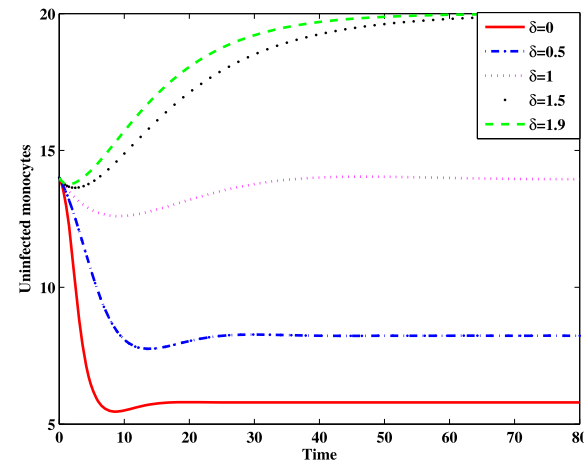

(a) Uninfected monocytes.

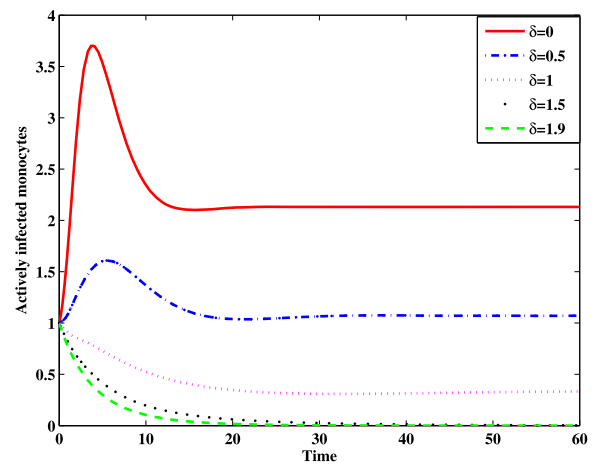

(c) Actively infected monocytes.

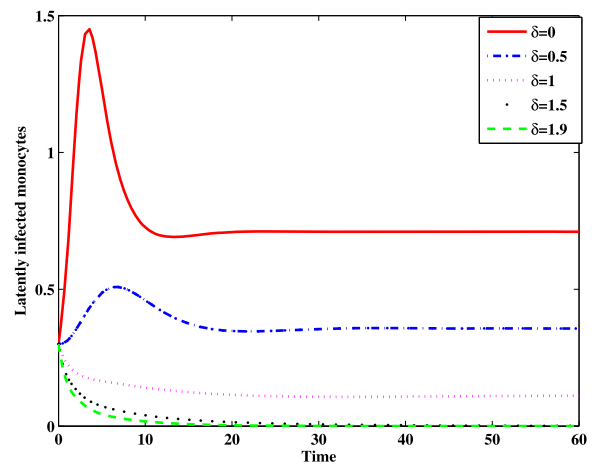

(b) Latently infected monocytes.

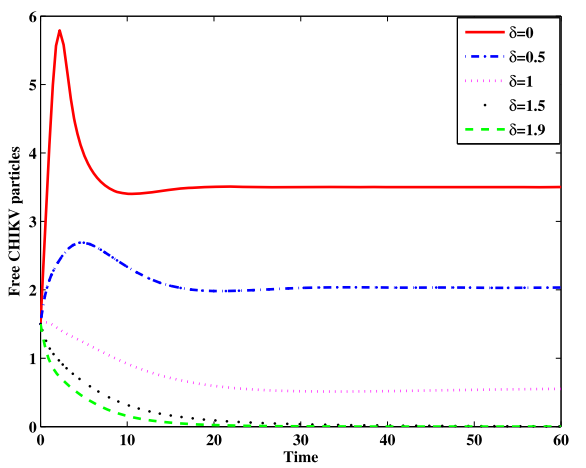

(d) Free CHIKV particles.

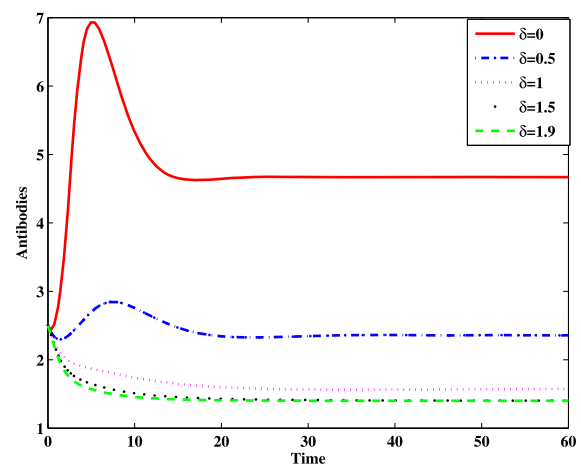

(e) Antibodies.

Figure 2 The simulation of trajectories of system (5)-(9) for Case 1

Table 2 The value of $\mathcal{R}_{0}$ for different values of $\tau$

\begin{tabular}{lll}
\hline$\delta$ & Equilibria & $\mathcal{R}_{0}$ \\
\hline 0.0 & $(5.79,0.71,2.13,3.50,4.67)$ & 9.0 \\
0.5 & $(8.22,0.35,1.07,2.03,2.35)$ & 3.6689 \\
1.0 & $(13.94,0.11,0.33,0.55,1.57)$ & 1.5668 \\
1.278 & $(20.00,0.00,0.00,0.00,1.40)$ & 1.00 \\
1.5 & $(20.00,0.00,0.00,0.00,1.40)$ & 0.7081 \\
2 & $(20.00,0.00,0.00,0.00,1.40)$ & 0.3404 \\
2.5 & $(20.00,0.00,0.00,0.00,1.40)$ & 0.1737 \\
\hline
\end{tabular}

(i) if $0 \leq \delta<1.278$, then $\Omega_{1}$ exists and it is G.A.S.;

(ii) if $\delta \geq 1.278$, then $\Omega_{0}$ is G.A.S. 
It is clearly seen that an increase in time delay will stabilize the system around $\Omega_{0}$. Biologically, the time delay has similar effect as the antiviral treatment, which can be used to eliminate the CHIKV. We observe that when the delay period is sufficiently long, the CHIKV replication will be cleared.

Case 2 We fix the value $\omega_{1}=\omega_{2}=0.05$. Let us consider $\mu=\mu_{1}=\mu_{2}$ and the initial

$$
\begin{array}{ll}
\vartheta_{1}(\varphi)=16, & \vartheta_{2}(\varphi)=0.05, \quad \vartheta_{3}(\varphi)=0.2, \\
\vartheta_{4}(\varphi)=0.3, & \vartheta_{5}(\varphi)=1.5, \quad \varphi \in[-k, 0] .
\end{array}
$$

The effect of the saturation on the CHIKV is shown in Fig. 3. It is shown that, as $\mu$ is increased, $s(t)$ is increased (but does not reach $s_{0}$ ) while all of $w(t), y(t), p(t)$, and $x(t)$ are decreased (but do not reach zero).

\section{Conclusion}

In the literature, most of the published papers which proposed within-host CHIKV dynamics models assumed that the susceptible monocyte becomes infected by contacting with CHIKV (CHIKV-to-monocyte transmission). However, it was reported that the $\mathrm{CHIKV}$ can also spread by infected-to-monocyte transmission. In this paper, we formulated and analyzed within-host CHIKV dynamics models with latently infected cells and antibody immune response. We incorporated both CHIKV-to-monocyte and infectedto-monocyte transmissions. We assumed that the infection rate of modeling CHIKV infection is given by saturated mass action. We incorporated three types of discrete or distributed time delays into the model. To ensure the well-posedness of the model, we proved the nonnegativity and boundedness properties of the solutions. We derived the basic reproduction number $\mathcal{R}_{0}$, which fully determines the existence and stability of the two equilibria of the model. The global stability of the equilibria of the model has been investigated by utilizing Lyapunov functionals and applying LaSalle's invariance principle. We have proven that (i) if $\mathcal{R}_{0} \leq 1$, then the CHIKV-free equilibrium $\Omega_{0}$ is globally asymptotically stable and the CHIKV is prophesied to be completely cleared from the body, and (ii) if $\mathcal{R}_{0}>$ 1 , then the infected equilibrium $\Omega_{1}$ is globally asymptotically stable and the CHIKV infection becomes chronic. We supported our theoretical results with numerical simulations.

Recently, many authors argued that the virus moves freely in body and follows the Fickian diffusion (see, e.g., [59] and [60]). Therefore, it is more reasonable to study reactiondiffusion versions of our models. Further, since the exact solutions of systems (5)-(9) and (17)-(21) are not known, therefore approximate solutions can only be found. Therefore, the corresponding discrete-time models of these systems need to be investigated (see, e.g., [61-63]). We leave these points as possible future works.

\section{Appendix}

Proof of Lemma 2 Let $\Omega(s, w, y, p, x)$ be any equilibrium satisfying:

$$
\begin{aligned}
& 0=\varrho-\xi s-\frac{\omega_{1} s p}{1+\mu_{1} p}-\frac{\omega_{2} s y}{1+\mu_{2} y}, \\
& 0=(1-n) e^{-a_{1} \delta_{1}}\left[\frac{\omega_{1} s p}{1+\mu_{1} p}+\frac{\omega_{2} s y}{1+\mu_{2} y}\right]-(b+d) w,
\end{aligned}
$$




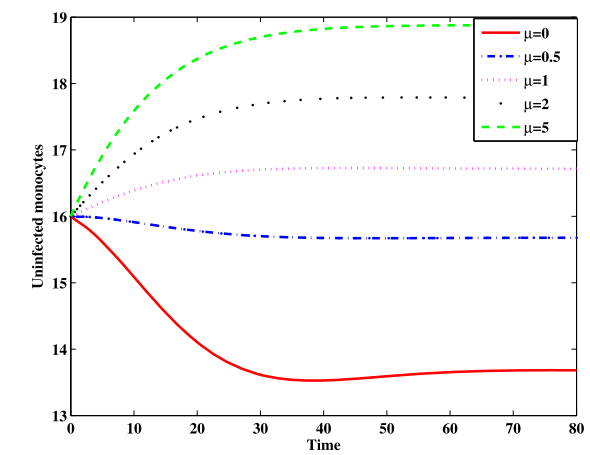

(a) Uninfected monocytes.

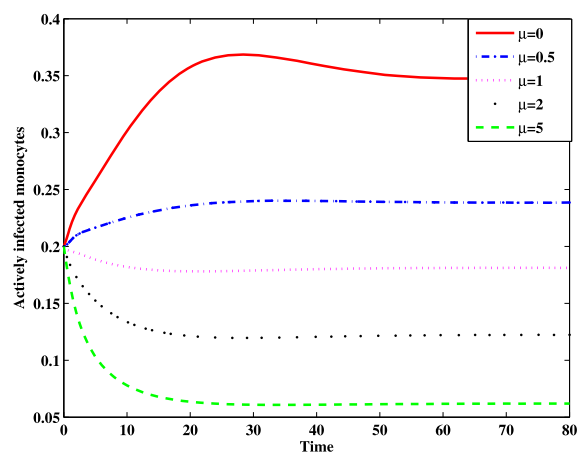

(c) Actively infected monocytes.

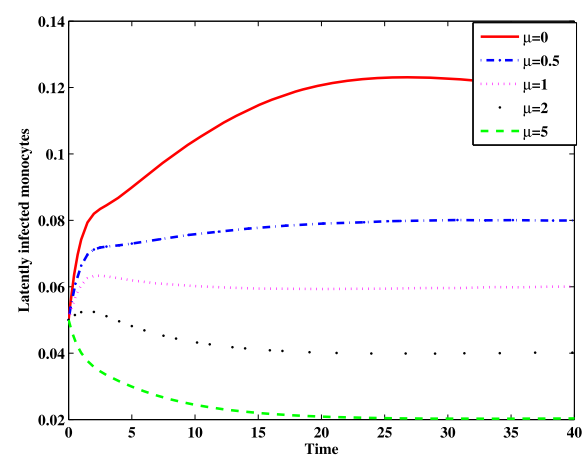

(b) Latently infected monocytes.

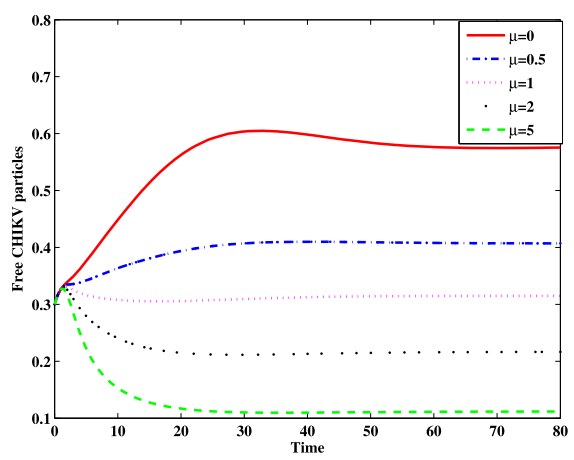

(d) Free CHIKV particles.

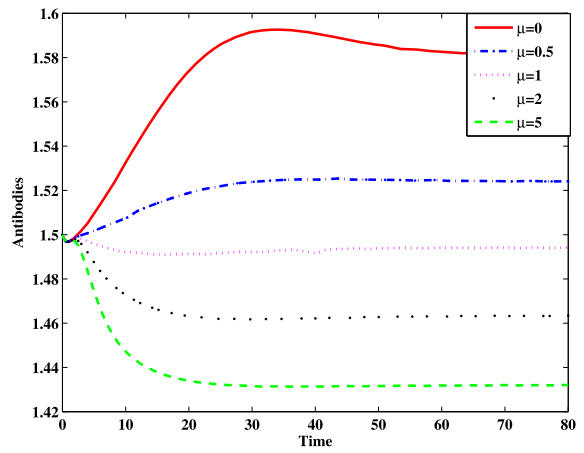

(e) Antibodies.

Figure 3 The simulation of trajectories of system (5)-(9) for Case 2

$$
\begin{aligned}
& 0=n e^{-a_{2} \delta_{2}}\left[\frac{\omega_{1} s p}{1+\mu_{1} p}+\frac{\omega_{2} s y}{1+\mu_{2} y}\right]+d w-\epsilon y, \\
& 0=\varkappa e^{-a_{3} \delta_{3}} y-c p-r x p \\
& 0=\lambda+\rho x p-m x .
\end{aligned}
$$

Solving Eqs. (25)-(29), we obtain CHIKV-free equilibrium $\Omega_{0}=\left(s_{0}, 0,0,0, x_{0}\right)$, where $s_{0}=$ $\frac{\varrho}{\xi}$ and $x_{0}=\frac{\lambda}{m}$. Moreover, we have

$$
\frac{D_{1} p^{4}+D_{2} p^{3}+D_{3} p^{2}+D_{4} p+D_{5}}{\bar{D}_{1} p^{4}+\bar{D}_{2} p^{3}+\bar{D}_{3} p^{2}+\bar{D}_{4} p+\bar{D}_{5}}=0
$$


where

$$
\begin{aligned}
& D_{1}=-(b+d) e^{a_{3} \delta_{3}} c^{2} \epsilon \rho^{2}\left(\mu_{1} \mu_{2} \xi+\mu_{2} \omega_{1}+\mu_{1} \omega_{2}\right), \\
& D_{2}=D_{21}+D_{22}+D_{23}+D_{24}+D_{25}+D_{26}+D_{27}+D_{28}, \\
& D_{3}=D_{31}+D_{32}+D_{33}+D_{34}+D_{35}+D_{36}+D_{37}+D_{38}+D_{39}+D_{310}, \\
& D_{4}=D_{41}+D_{42}+D_{43}+D_{44}+D_{45}+D_{46}+D_{47}+D_{48}, \\
& D_{5}=D_{51}+D_{52}+D_{53}+D_{54}, \\
& C_{1}=c(b+d) e^{a_{3} \delta_{3}} \varkappa \rho^{2}\left(\mu_{1} \mu_{2} \xi+\mu_{2} \omega_{1}+\mu_{1} \omega_{2}\right), \\
& C_{2}=C_{21}+C_{22}, \\
& C_{3}=C_{31}+C_{32}+C_{33}, \\
& C_{4}=(b+d) m \varkappa\left(-2 \varkappa \xi \rho+m \varkappa\left(\mu_{1} \xi+\omega_{1}\right)+e^{a_{3} \delta_{3}}(c m+r \lambda)\left(\mu_{2} \xi+\omega_{2}\right)\right), \\
& C_{5}=(b+d) m^{2} \varkappa^{2} \xi,
\end{aligned}
$$

and

$$
\begin{aligned}
& D_{21}=c \rho^{2} b \varkappa\left\{-\epsilon\left(\mu_{1} \xi+\omega_{1}\right)+e^{-a_{2} \delta_{2}} n \varrho\left(\mu_{2} \omega_{1}+\mu_{1} \omega_{2}\right)\right\}, \\
& D_{22}=2 c \rho b e^{a_{3} \delta_{3}} \epsilon_{2} r \lambda\left\{\mu_{2} \omega_{1}+\mu_{1}\left(\mu_{2} \xi+\omega_{2}\right)\right\} \text {, } \\
& D_{23}=c^{2} \rho b e^{a_{3} \delta_{3}} \epsilon\left\{-\rho\left(\mu_{2} \xi+\omega_{2}\right)+2 m\left(\mu_{1} \mu_{2} \xi+\mu_{2} \omega_{1}+\mu_{1} \omega_{2}\right)\right\} \text {, } \\
& D_{24}=c \rho^{2} d e^{-a_{2} \delta_{2}} n \varkappa \varrho\left(\mu_{2} \omega_{1}+\mu_{1} \omega_{2}\right) \text {, } \\
& D_{25}=2 e^{a_{3} \delta_{3}} c \rho d r \epsilon \lambda\left\{\mu_{2} \omega_{1}+\mu_{1}\left(\mu_{2} \xi+\omega_{2}\right)\right\} \text {, } \\
& D_{26}=c \rho^{2} d \varkappa\left\{-\epsilon\left(\mu_{1} \xi+\omega_{1}\right)-e^{-a_{1} \delta_{1}}(-1+n) \varrho\left(\mu_{2} \omega_{1}+\mu_{1} \omega_{2}\right)\right\} \text {, } \\
& D_{27}=-c^{2} \rho^{2} d e^{a_{3} \delta_{3}} \epsilon\left(\mu_{2} \xi+\omega_{2}\right), \\
& D_{28}=2 m c^{2} \rho d e^{a_{3} \delta_{3}} \epsilon\left(\mu_{1} \mu_{2} \xi+\mu_{2} \omega_{1}+\mu_{1} \omega_{2}\right), \\
& D_{31}=\varkappa^{2} \rho^{2} \varrho \omega_{1}\left\{-d e^{-a_{1} \delta_{1}-a_{3} \delta_{3}}(-1+n)+(b+d) e^{-a_{2} \delta_{2}-a_{3} \delta_{3}} n\right\}, \\
& D_{32}=b \varkappa r \in \lambda \rho\left(\mu_{1} \xi+\omega_{1}\right)+d \varkappa r \in \lambda \rho\left(\mu_{1} \xi+\omega_{1}\right) \text {, } \\
& D_{33}=b c \varkappa \epsilon \rho\left\{-\xi \rho+2 m\left(\mu_{1} \xi+\omega_{1}\right)\right\}+c d \varkappa \epsilon \rho\left\{-\xi \rho+2 m\left(\mu_{1} \xi+\omega_{1}\right)\right\}, \\
& D_{34}=d e^{-a_{1} \delta_{1}}(-1+n) \varkappa r \lambda \rho \varrho\left(\mu_{2} \omega_{1}+\mu_{1} \omega_{2}\right) \text {, } \\
& D_{35}=-(b+d) e^{-a_{2} \delta_{2}} n \varkappa r \lambda \rho \varrho\left(\mu_{2} \omega_{1}+\mu_{1} \omega_{2}\right) \text {, } \\
& D_{36}=(b+d) c e^{-a_{2} \delta_{2}} n \varkappa \rho \varrho\left\{\rho \omega_{2}-2 m\left(\mu_{2} \omega_{1}+\mu_{1} \omega_{2}\right)\right\}, \\
& D_{37}=c d e^{-a_{1} \delta_{1}}(-1+n) \varkappa \rho \varrho\left\{-\rho \omega_{2}+2 m\left(\mu_{2} \omega_{1}+\mu_{1} \omega_{2}\right)\right\} \text {, } \\
& D_{38}=-(b+d) e^{a_{3} \delta_{3}} \epsilon(c m+r \lambda)\left\{r \lambda \mu_{2} \omega_{1}+r \lambda \mu_{1}\left(\mu_{2} \xi+\omega_{2}\right)\right\}, \\
& D_{39}=2(b+d) e^{a_{3} \delta_{3}} \epsilon(c m+r \lambda) c \rho\left(\mu_{2} \xi+\omega_{2}\right), \\
& D_{310}=-(b+d) e^{a_{3} \delta_{3}} \epsilon(c m+r \lambda) c m\left\{\mu_{2} \omega_{1}+\mu_{1}\left(\mu_{2} \xi+\omega_{2}\right)\right\}, \\
& D_{41}=2 m\left\{d e^{-a_{1} \delta_{1}-a_{3} \delta_{3}}(-1+n)-(b+d) e^{-a_{2} \delta_{2}-a_{3} \delta_{3}} n\right\} \varkappa^{2} \rho \varrho \omega_{1},
\end{aligned}
$$




$$
\begin{aligned}
& D_{42}=(b+d) \varkappa r \epsilon\left\{\xi \rho-m\left(\mu_{1} \xi+\omega_{1}\right)\right\} \\
& D_{43}=-(b+d) c m \varkappa \epsilon\left\{-2 \xi \rho+m\left(\mu_{1} \xi+\omega_{1}\right)\right\}, \\
& D_{44}=-(b+d) e^{a_{3} \delta_{3}} \epsilon(c m+r \lambda)^{2}\left(\mu_{2} \xi+\omega_{2}\right), \\
& D_{45}=-c d e^{-a_{1} \delta_{1}} m(-1+n) \varkappa \varrho\left(m \mu_{2} \omega_{1}+m \mu_{1} \omega_{2}-2 \rho \omega_{2}\right), \\
& D_{46}=(b+d) c e^{-a_{2} \delta_{2}} m n \varkappa \varrho\left(m \mu_{2} \omega_{1}+m \mu_{1} \omega_{2}-2 \rho \omega_{2}\right), \\
& D_{47}=-d e^{-a_{1} \delta_{1}}(-1+n) \varkappa r \lambda \varrho\left(m \mu_{2} \omega_{1}+m \mu_{1} \omega_{2}-\rho \omega_{2}\right), \\
& D_{48}=(b+d) e^{-a_{2} \delta_{2}} n \varkappa r \lambda \varrho\left(m \mu_{2} \omega_{1}+m \mu_{1} \omega_{2}-\rho \omega_{2}\right), \\
& D_{51}=e^{-a_{1} \delta_{1}-a_{2} \delta_{2}-a_{3} \delta_{3}} m^{2} \varkappa{ }^{2}\left\{-d e^{a_{2} \delta_{2}}(-1+n)+(b+d) e^{a_{1} \delta_{1}} n\right\} \varrho \omega_{1}, \\
& D_{52}=e^{-a_{1} \delta_{1}-a_{2} \delta_{2}-a_{3} \delta_{3}} m \varkappa\left\{e^{a_{3} \delta_{3}}[c m+r \lambda]\left[(b+d) e^{a_{1} \delta_{1}} n \varrho \omega_{2}\right]\right\} \\
& D_{53}=e^{-a_{1} \delta_{1}-a_{2} \delta_{2}-a_{3} \delta_{3}} m \varkappa\left\{e^{a_{3} \delta_{3}}[c m+r \lambda]\left[e^{a_{2} \delta_{2}}(b+d) e^{a_{1} \delta_{1}} \epsilon \xi\right]\right\}, \\
& D_{54}=e^{-a_{1} \delta_{1}-a_{2} \delta_{2}-a_{3} \delta_{3}} m \varkappa\left\{e^{a_{3} \delta_{3}}[c m+r \lambda]\left[e^{a_{2} \delta_{2}} d(-1+n) \varrho \omega_{2}\right]\right\}, \\
& C_{21}=(b+d) \varkappa \rho\left(\varkappa \rho\left(\mu_{1} \xi+\omega_{1}\right)-e^{a_{3} \delta_{3}} r \lambda\left(\mu_{1} \mu_{2} \xi+\mu_{2} \omega_{1}+\mu_{1} \omega_{2}\right)\right), \\
& C_{22}=(b+d) \varkappa \rho\left(-c e^{a_{3} \delta_{3}}\left(-\rho\left(\mu_{2} \xi+\omega_{2}\right)+2 m\left(\mu_{1} \mu_{2} \xi+\mu_{2} \omega_{1}+\mu_{1} \omega_{2}\right)\right)\right), \\
& C_{31}=(b+d) \varkappa\left(\varkappa \rho\left(\xi \rho-2 m\left(\mu_{1} \xi+\omega_{1}\right)\right)\right), \\
& C_{32}=(b+d) \varkappa\left(c e^{a_{3} \delta_{3}} m\left(m \mu_{2} \omega_{1}+m \mu_{1}\left(\mu_{2} \xi+\omega_{2}\right)-2 \rho\left(\mu_{2} \xi+\omega_{2}\right)\right)\right), \\
& C_{33}=(b+d) \varkappa\left(e^{a_{3} \delta_{3}} r \lambda\left(m \mu_{2} \omega_{1}+m \mu_{1}\left(\mu_{2} \xi+\omega_{2}\right)-\rho\left(\mu_{2} \xi+\omega_{2}\right)\right)\right) .
\end{aligned}
$$

Let us define a function $X_{1}(p)$ as follows:

$$
X_{1}(p)=\frac{D_{1} p^{4}+D_{2} p^{3}+D_{3} p^{2}+D_{4} p+D_{5}}{C_{1} p^{4}+C_{2} p^{3}+C_{3} p^{2}+C_{4} p+C_{5}}=0 .
$$

Then we obtain

$$
\begin{gathered}
X_{1}(0)=\frac{e^{a_{3} \delta 3} \epsilon(c m+r \lambda)\left(\mathcal{R}_{0}-1\right)}{m \varkappa}, \\
\lim _{p \rightarrow\left(\frac{m}{\rho}\right)^{-}} X_{1}(p)=-\frac{e^{a_{3} \delta 3} r \epsilon \lambda}{\rho \varkappa}<0 .
\end{gathered}
$$

Therefore, if $\mathcal{R}_{0}>1$, then $X_{1}(0)>0$, and there exists $p_{1} \in\left(0, \frac{m}{\rho}\right)$ such that $X_{1}\left(p_{1}\right)=0$. It follows that

$$
\begin{aligned}
& x_{1}=\frac{\lambda}{m-\rho p_{1}}>0, \\
& y_{1}=\frac{p_{1}\left(c+r x_{1}\right)}{e^{-a_{3} \delta_{3} \varkappa}>0,} \\
& s_{1}=\frac{\varrho}{\xi+\frac{\omega_{1} p_{1}}{1+\mu_{1} p_{1}}+\frac{\omega_{2} y_{1}}{1+\mu_{2} y_{1}}}>0, \\
& w_{1}=\frac{(1-n) e^{-a_{1} \delta_{1}}}{b+d}\left[\frac{\omega_{1} s_{1} p_{1}}{1+\mu_{1} p_{1}}+\frac{\omega_{2} s_{1} y_{1}}{1+\mu_{2} y_{1}}\right]>0 .
\end{aligned}
$$

Thus, if $\mathcal{R}_{0}>1$, then the system has an infected equilibrium $\Omega_{1}=\left(s_{1}, w_{1}, y_{1}, p_{1}, x_{1}\right)$. 
Proof of Lemma 4 Let $\Omega(s, w, y, p, x)$ be any equilibrium satisfying:

$$
\begin{aligned}
& 0=\varrho-\xi s-\frac{\omega_{1} s p}{1+\mu_{1} p}-\frac{\omega_{2} s y}{1+\mu_{2} y}, \\
& 0=(1-n) F_{1}\left[\frac{\omega_{1} s p}{1+\mu_{1} p}+\frac{\omega_{2} s y}{1+\mu_{2} y}\right]-(b+d) w, \\
& 0=n F_{2}\left[\frac{\omega_{1} s p}{1+\mu_{1} p}+\frac{\omega_{2} s y}{1+\mu_{2} y}\right]+d w-\epsilon y, \\
& 0=\varkappa F_{3} y-c p-r x p \\
& 0=\lambda+\rho x p-m x .
\end{aligned}
$$

Solving Eqs. (30)-(34), then there exists a CHIKV-free equilibrium $\Omega_{0}=\left(s_{0}, 0,0,0, x_{0}\right)$, where $s_{0}=\frac{\varrho}{\xi}$ and $x_{0}=\frac{\lambda}{m}$. From Eqs. (30)-(34) we have

$$
\begin{aligned}
& s=\frac{\varrho}{\xi+\frac{\omega_{1} p}{1+\mu_{1} p}+\frac{\omega_{2} y}{1+\mu_{2} y},} \\
& w=\frac{(1-n) F_{1} s\left(\omega_{1} p+\omega_{1} p \mu_{2} y+\omega_{2} y+\omega_{2} y \mu_{1} p\right)}{(b+d)\left(1+\mu_{1} p\right)\left(1+\mu_{2} y\right)}, \\
& y=\frac{p(c+r x)}{F_{3} \varkappa}, \\
& x=\frac{\lambda}{m-\rho p} .
\end{aligned}
$$

Substituting from Eqs. (35)-(38) into (32), we get

$$
\frac{\bar{D}_{1} p^{4}+\bar{D}_{2} p^{3}+\bar{D}_{3} p^{2}+\bar{D}_{4} p+\bar{D}_{5}}{\bar{C}_{1} p^{4}+\bar{C}_{2} p^{3}+\bar{C}_{3} p^{2}+\bar{C}_{4} p+\bar{C}_{5}}=0
$$

where

$$
\begin{aligned}
\bar{D}_{1}= & -(b+d) c^{2} \epsilon \rho^{2}\left\{\mu_{2} \omega_{1}+\mu_{1}\left(\mu_{2} \xi+\omega_{2}\right)\right\}, \\
\bar{D}_{2}= & \bar{D}_{21}+\bar{D}_{22}+\bar{D}_{23}+\bar{D}_{24}+\bar{D}_{25}+\bar{D}_{26}+\bar{D}_{27}+\bar{D}_{28}+\bar{D}_{29}, \\
\bar{D}_{3}= & \bar{D}_{31}+\bar{D}_{32}+\bar{D}_{33}+\bar{D}_{34}+\bar{D}_{35}+\bar{D}_{36}+\bar{D}_{37}+\bar{D}_{38}+\bar{D}_{39}+\bar{D}_{310}+\bar{D}_{311} \\
& +\bar{D}_{312}+\bar{D}_{313}+\bar{D}_{314}, \\
\bar{D}_{4}= & \bar{D}_{41}+\bar{D}_{42}+\bar{D}_{43}+\bar{D}_{44}+\bar{D}_{45}+\bar{D}_{46}+\bar{D}_{47}+\bar{D}_{48}+\bar{D}_{49}+\bar{D}_{410}+\bar{D}_{411} \\
& +\bar{D}_{412}+\bar{D}_{413}+\bar{D}_{414}+\bar{D}_{415}+\bar{D}_{416}+\bar{D}_{417}+\bar{D}_{418}+\bar{D}_{419}, \\
\bar{D}_{5}= & \bar{D}_{51}+\bar{D}_{52}+\bar{D}_{53}+\bar{D}_{54}, \\
\bar{C}_{1}= & (b+d) F_{3} \varkappa \rho\left(c \mu_{1} \mu_{2} \xi \rho+c \mu_{2} \rho \omega_{1}+c \mu_{1} \rho \omega_{2}\right), \\
\bar{C}_{2}= & \bar{C}_{21}+\bar{C}_{22}, \\
\bar{C}_{3}= & \bar{C}_{31}+\bar{C}_{32}+\bar{C}_{33}, \\
\bar{C}_{4}= & (b+d) F_{3} m \varkappa\left(F_{3} \varkappa\left(-2 \xi \rho+m\left(\mu_{1} \xi+\omega_{1}\right)\right)+(c m+r \lambda)\left(\mu_{2} \xi+\omega_{2}\right)\right),
\end{aligned}
$$




$$
\bar{C}_{5}=(b+d) F_{3}^{2} m^{2} \varkappa^{2} \xi,
$$

and

$$
\begin{aligned}
& \bar{D}_{21}=2 c \rho b r \epsilon \lambda\left\{\mu_{2} \omega_{1}+\mu_{1}\left(\mu_{2} \xi+\omega_{2}\right)\right\} \text {, } \\
& \bar{D}_{22}=c \rho b F_{3} \varkappa \rho\left\{-\epsilon\left(\mu_{1} \xi+\omega_{1}\right)+F_{2} n \varrho\left(\mu_{2} \omega_{1}+\mu_{1} \omega_{2}\right)\right\} \text {, } \\
& \bar{D}_{23}=-c^{2} \rho^{2} b \epsilon\left(\mu_{2} \xi+\omega_{2}\right) \text {, } \\
& \bar{D}_{24}=2 c^{2} \rho b m \epsilon\left(\mu_{1} \mu_{2} \xi+\mu_{2} \omega_{1}+\mu_{1} \omega_{2}\right), \\
& \bar{D}_{25}=2 c \rho d r \epsilon \lambda\left\{\mu_{2} \omega_{1}+\mu_{1}\left(\mu_{2} \xi+\omega_{2}\right)\right\} \text {, } \\
& \bar{D}_{26}=-c \rho^{2} d F_{3} \varkappa \epsilon\left(\mu_{1} \xi+\omega_{1}\right) \text {, } \\
& \bar{D}_{27}=-c \rho^{2} d F_{3} \varkappa\left\{F_{1}(-1+n)-F_{2} n\right\} \varrho\left(\mu_{2} \omega_{1}+\mu_{1} \omega_{2}\right), \\
& \bar{D}_{28}=-c^{2} \rho^{2} d \epsilon\left(\mu_{2} \xi+\omega_{2}\right) \text {, } \\
& \bar{D}_{29}=2 c^{2} \rho d \epsilon m\left(\mu_{1} \mu_{2} \xi+\mu_{2} \omega_{1}+\mu_{1} \omega_{2}\right), \\
& \bar{D}_{31}=-b\left(r \lambda \mu_{2}-F_{3} \varkappa \rho\right)\left\{F_{2} F_{3} n \varkappa \rho \varrho \omega_{1}+r \in \lambda\left(\mu_{1} \xi+\omega 1\right)\right\}, \\
& \bar{D}_{32}=-b r \lambda \mu_{1}\left(r \in \lambda+F_{2} F_{3} n \varkappa \rho \varrho\right) \omega_{2} \text {, } \\
& \bar{D}_{33}=-b c^{2} m \epsilon\left\{m \mu_{2} \omega_{1}+m \mu_{1}\left(\mu_{2} \xi+\omega_{2}\right)-2 \rho\left(\mu_{2} \xi+\omega_{2}\right)\right\} \text {, } \\
& \bar{D}_{34}=-b c \rho\left\{-2 r \epsilon \lambda\left(\mu_{2} \xi+\omega_{2}\right)+F_{3} \varkappa \rho\left(\epsilon \xi-F_{2} n \varrho \omega_{2}\right)\right\} \text {, } \\
& \bar{D}_{35}=-2 b c m r \in \lambda\left\{\mu_{2} \omega_{1}+\mu_{1}\left(\mu_{2} \xi+\omega_{2}\right)\right\} \text {, } \\
& \bar{D}_{36}=-2 b c m F_{3} \varkappa \rho\left\{-\epsilon\left(\mu_{1} \xi+\omega_{1}\right)+F_{2} n \varrho\left(\mu_{2} \omega_{1}+\mu_{1} \omega_{2}\right)\right\} \text {, } \\
& \bar{D}_{37}=-d\left(r \lambda \mu_{2}-F_{3} \varkappa \rho\right)\left\{F_{3}\left(F_{1}-F_{1} n+F_{2} n\right) \varkappa \rho \varrho \omega_{1}+r \in \lambda\left(\mu_{1} \xi+\omega_{1}\right)\right\} \text {, } \\
& \bar{D}_{38}=-d r \lambda \mu_{1} \omega_{2}\left\{r \in \lambda+F_{3}\left(F_{1}-F_{1} n+F_{2} n\right) \varkappa \rho \varrho\right\} \text {, } \\
& \bar{D}_{39}=-d c^{2} m \epsilon\left\{m \mu_{2} \omega_{1}+m \mu_{1}\left(\mu_{2} \xi+\omega_{2}\right)-2 \rho\left(\mu_{2} \xi+\omega_{2}\right)\right\}, \\
& \bar{D}_{310}=2 d c \rho r \epsilon \lambda\left(\mu_{2} \xi+\omega_{2}\right) \text {, } \\
& \bar{D}_{311}=-d c \rho^{2} F_{3} \varkappa\left\{\epsilon \xi+\left(F_{1}(-1+n)-F_{2} n\right) \varrho \omega_{2}\right\}, \\
& \bar{D}_{312}=-2 d c m r \epsilon \lambda\left\{\mu_{2} \omega_{1}+\mu_{1}\left(\mu_{2} \xi+\omega_{2}\right)\right\} \text {, } \\
& \bar{D}_{313}=2 d c m F_{3} \varkappa \rho \epsilon\left(\mu_{1} \xi+\omega_{1}\right) \text {, } \\
& \bar{D}_{314}=2 d c m F_{3} \varkappa \rho\left\{F_{1}(-1+n)-F_{2} n\right\} \varrho\left(\mu_{2} \omega_{1}+\mu_{1} \omega_{2}\right) \text {, } \\
& \bar{D}_{41}=-2 b F_{2} F_{3}^{2} m n \varkappa^{2} \rho \varrho \omega_{1} \text {, } \\
& \bar{D}_{42}=-b c^{2} m^{2} \epsilon\left(\mu_{2} \xi+\omega_{2}\right) \text {, } \\
& \bar{D}_{43}=-b r^{2} \epsilon \lambda^{2}\left(\mu_{2} \xi+\omega_{2}\right) \text {, } \\
& \bar{D}_{44}=-b F_{3} \varkappa r \lambda\left\{-\epsilon \xi \rho+m \epsilon\left(\mu_{1} \xi+\omega_{1}\right)\right\} \text {, } \\
& \bar{D}_{45}=-b F_{3} \varkappa r \lambda F_{2} n \rho \varrho \omega_{2} \text {, } \\
& \bar{D}_{46}=b F_{3} \varkappa r \lambda F_{2} m n \varrho\left(\mu_{2} \omega_{1}+\mu_{1} \omega_{2}\right) \text {, } \\
& \bar{D}_{47}=-2 b c m r \epsilon \lambda\left(\mu_{2} \xi+\omega_{2}\right) \text {, }
\end{aligned}
$$




$$
\begin{aligned}
& \bar{D}_{48}=2 b c m F_{3} \varkappa \epsilon \xi \rho, \\
& \bar{D}_{49}=-b c m F_{3} \varkappa\left\{m \epsilon\left(\mu_{1} \xi+\omega_{1}\right)+2 F_{2} n \rho \varrho \omega_{2}\right\}, \\
& \bar{D}_{410}=b c m F_{3} \varkappa F_{2} m n \varrho\left(\mu_{2} \omega_{1}+\mu_{1} \omega_{2}\right), \\
& \bar{D}_{411}=-2 d F_{3}^{2} m\left(F_{1}-F_{1} n+F_{2} n\right) \varkappa^{2} \rho \varrho \omega_{1}, \\
& \bar{D}_{412}=-d c^{2} m^{2} \epsilon\left(\mu_{2} \xi+\omega_{2}\right)-d r^{2} \epsilon \lambda^{2}\left(\mu_{2} \xi+\omega_{2}\right), \\
& \bar{D}_{413}=-d F_{3} \varkappa r \lambda\left\{-\epsilon \xi \rho+m \epsilon\left(\mu_{1} \xi+\omega_{1}\right)\right\}, \\
& \bar{D}_{414}=-d F_{3} \varkappa r \lambda\left(F_{1}-F_{1} n+F_{2} n\right) \rho \varrho \omega_{2}, \\
& \bar{D}_{415}=-d F_{3} \varkappa r \lambda m\left\{F_{1}(-1+n)-F_{2} n\right\} \varrho\left(\mu_{2} \omega_{1}+\mu_{1} \omega_{2}\right), \\
& \bar{D}_{416}=-2 d c m r \epsilon \lambda\left(\mu_{2} \xi+\omega_{2}\right), \\
& \bar{D}_{417}=-d c m F_{3} \varkappa\left\{-2 \epsilon \xi \rho+m \epsilon\left(\mu_{1} \xi+\omega_{1}\right)\right\}, \\
& \bar{D}_{418}=-2 d c m F_{3} \varkappa\left(F_{1}-F_{1} n+F_{2} n\right) \rho \varrho \omega_{2}, \\
& \bar{D}_{419}=-d c m F_{3} \varkappa m\left\{F_{1}(-1+n)-F_{2} n\right\} \varrho\left(\mu_{2} \omega_{1}+\mu_{1} \omega_{2}\right), \\
& \bar{D}_{51}=F_{3} m \varkappa(-b c m \epsilon \xi-c d m \epsilon \xi-b r \epsilon \lambda \xi-d r \epsilon \lambda \xi), \\
& \bar{D}_{52}=F_{3} m \varkappa\left(d F_{1} F_{3} m \varkappa \varrho \omega_{1}-d F_{1} F_{3} m n \varkappa \varrho \omega_{1}\right), \\
& \bar{D}_{53}=F_{3} m \varkappa\left(b F_{2} F_{3} m n \varkappa \varrho \omega_{1}+d F_{2} F_{3} m n \varkappa \varrho \omega_{1}\right), \\
& \bar{D}_{54}=F_{3} m \varkappa\left\{\left[b F_{2} n+d\left(F_{1}-F_{1} n+F_{2} n\right)\right](c m+r \lambda) \varrho \omega_{2}\right\}, \\
& \bar{C}_{21}=(b+d) F_{3} \varkappa \rho\left(\left(-r \lambda \mu_{2}+F_{3} \varkappa \rho\right)\left(\mu_{1} \xi+\omega_{1}\right)-r \lambda \mu_{1} \omega_{2}\right), \\
& \bar{C}_{22}=(b+d) F_{3} \varkappa \rho\left(c \rho\left(\mu_{2} \xi+\omega_{2}\right)-2 c m\left(\mu_{2} \omega_{1}+\mu_{1}\left(\mu_{2} \xi+\omega_{2}\right)\right)\right), \\
& \bar{C}_{31}=(b+d) F_{3} \varkappa\left(m\left(r \lambda \mu_{2}-2 F_{3} \varkappa \rho\right)\left(\mu_{1} \xi+\omega_{1}\right)\right), \\
& \bar{C}_{33}=(b+d) F_{3} \varkappa\left(c m\left(m \mu_{2} \omega_{1}+m \mu_{1}\left(\mu_{2} \xi+\omega_{2}\right)-2 \rho\left(\mu_{2} \xi+\omega_{2}\right)\right)\right) .
\end{aligned}
$$

Let us define a function $X_{2}(p)$ as follows:

$$
X_{2}(p)=\frac{\bar{D}_{1} p^{4}+\bar{D}_{2} p^{3}+\bar{D}_{3} p^{2}+\bar{D}_{4} p+\bar{D}_{5}}{\bar{C}_{1} p^{4}+\bar{C}_{2} p^{3}+\bar{C}_{3} p^{2}+\bar{C}_{4} p+\bar{C}_{5}}=0
$$

Then we obtain

$$
\begin{gathered}
X_{2}(0)=\frac{\epsilon(c m+r \lambda)\left(\mathcal{R}_{0}^{D}-1\right)}{F_{3} m \varkappa}, \\
\lim _{p \rightarrow\left(\frac{m}{\rho}\right)^{-}} X_{1}(p)=-\frac{r \epsilon \lambda}{F_{3} \rho \varkappa}<0 .
\end{gathered}
$$

Therefore, if $\mathcal{R}_{0}^{D}>1$, then $X_{2}(0)>0$ and $\exists p_{1} \in\left(0, \frac{m}{\rho}\right)$ such that $X_{2}\left(p_{1}\right)=0$. If $\mathcal{R}_{0}^{D}>1$, then Eqs. (35)-(38) yield

$$
x_{1}=\frac{\lambda}{m-\rho p_{1}}>0
$$




$$
\begin{aligned}
& y_{1}=\frac{p_{1}\left(c+r x_{1}\right)}{F_{3} \varkappa}>0, \\
& s_{1}=\frac{\varrho}{\xi+\frac{\omega_{1} p_{1}}{1+\mu_{1} p_{1}}+\frac{\omega_{2} y_{1}}{1+\mu_{2} y_{1}}}>0, \\
& w_{1}=\frac{(1-n) F_{1}}{b+d}\left[\frac{\omega_{1} s_{1} p_{1}}{1+\mu_{1} p_{1}}+\frac{\omega_{2} s_{1} y_{1}}{1+\mu_{2} y_{1}}\right]>0,
\end{aligned}
$$

and $\Omega_{1}\left(s_{1}, w_{1}, y_{1}, p_{1}, x_{1}\right)$ exists.

\section{Funding}

This project was funded by the Deanship of Scientific Research (DSR), King Abdulaziz University, Jeddah, under grant No. (DF-007-130-1441). The authors, therefore, gratefully acknowledge DSR technical and financial support.

\section{Availability of data and materials}

Not applicable.

\section{Competing interests}

The authors declare that they have no competing interests.

\section{Authors' contributions}

All authors contributed equally to the writing of this paper. All authors read and approved the final manuscript.

\section{Author details}

${ }^{1}$ Department of Mathematics, Faculty of Science, King Abdulaziz University, Jeddah, Saudi Arabia. ${ }^{2}$ Jeddah College of Technology, Technical and Vocational Training Corporation, Jeddah, Saudi Arabia.

\section{Publisher's Note}

Springer Nature remains neutral with regard to jurisdictional claims in published maps and institutional affiliations.

\section{Received: 22 March 2019 Accepted: 6 November 2019 Published online: 15 November 2019}

\section{References}

1. Chitnis, N., Cushing, J.M., Hyman, J.M.: Bifurcation analysis of a mathematical model for malaria transmission. SIAM J. Appl. Math. 67(1), 24-45 (2006)

2. Mandal, S., Sarkar, R.R., Sinha, S.: Mathematical models of malaria-a review. Malar. J. 10, 202 (2011)

3. Beretta, E., Capasso, V., Garao, D.G.: A mathematical model for malaria transmission with asymptomatic carriers and two age groups in the human population. Math. Biosci. 300, 87-101 (2018)

4. Esteva, L., Vargas, C.: A model for dengue disease with variable human population. J. Math. Biol. 38, 220 (1999)

5. Abdelrazec, A., Belair, J., Shan, C.H., Zhu, H.P.: Modeling the spread and control of dengue with limited public health resources. Math. Biosci. 271, 136-145 (2016)

6. Zhu, M., Xu, Y.: A time-periodic dengue fever model in a heterogeneous environment. Math. Comput. Simul. 155, 115-129 (2019)

7. Bonyah, E., Okosun, K.O.: Mathematical modeling of Zika virus. Asian Pac. J. Trop. Dis. 6(9), 673-679 (2016)

8. Dantas, E., Tosin, M., Cunha, A. Jr.: Calibration of a SEIR-SEl epidemic model to describe the Zika virus outbreak in Brazil. Appl. Math. Comput. 338, 249-259 (2018)

9. Raimundo, S.M., Amaku, M., Massad, E.: Equilibrium analysis of a yellow fever dynamical model with vaccination. Comput. Math. Methods Med. 2015, Article ID 482091 (2015)

10. Dumont, Y., Chiroleu, F.: Vector control for the chikungunya disease. Math. Biosci. Eng. 7, 313-345 (2010)

11. Dumont, Y., Tchuenche, J.M.: Mathematical studies on the sterile insect technique for the chikungunya disease and aedes albopictus. J. Math. Biol. 65(5), 809-854 (2012)

12. Dumont, Y., Chiroleu, F., Domerg, C.: On a temporal model for the chikungunya disease: modeling, theory and numerics. Math. Biosci. 213, 80-91 (2008)

13. Manore, C.A., Hickmann, K.S., Xu, S., Wearing, H.J., Hyman, J.M.: Comparing dengue and chikungunya emergence and endemic transmission in A. aegypti and A. albopictus. J. Theor. Biol. 356, 174-191 (2014)

14. Liu, X., Stechlinski, P.: Application of control strategies to a seasonal model of chikungunya disease. Appl. Math. Model. 39, 3194-3220 (2015)

15. Nowak, M.A., Bangham, C.R.M.: Population dynamics of immune responses to persistent viruses. Science 272, 74-79 (1996)

16. Perelson, A.S., Essunger, P., Cao, Y., Vesanen, M., Hurley, A., Saksela, K., Markowitz, M., Ho, D.D.: Decay characteristics of HIV-1-infected compartments during combination therapy. Nature 387, 188-191 (1997)

17. Perelson, A.S., Nelson, P.W.: Mathematical analysis of HIV-1 dynamics in vivo. SIAM Rev. 41(1), 3-44 (1999)

18. Nowak, M.A., May, R.M.: Virus Dynamics: Mathematical Principles of Immunology and Virology. Oxford University Press, Oxford (2000)

19. Elaiw, A.M., Elnahary, E.Kh., Raezah, A.A.: Effect of cellular reservoirs and delays on the global dynamics of HIV. Adv. Differ. Equ. 2018, 85 (2018)

20. Wodarz, D., Nowak, M.A.: Mathematical models of HIV pathogenesis and treatment. BioEssays 24, 1178-1187 (2002) 
21. Elaiw, A.M., Almuallem, N.A.: Global dynamics of delay-distributed HIV infection models with differential drug efficacy in cocirculating target cells. Math. Methods Appl. Sci. 39, 4-31 (2016)

22. Elaiw, A.M., Almuallem, N.A.: Global properties of delayed-HIV dynamics models with differential drug efficacy in cocirculating target cells. Appl. Math. Comput. 265, 1067-1089 (2015)

23. Elaiw, A.M., AlShamrani, N.H.: Stability of a general adaptive immunity virus dynamics model with multi-stages of infected cells and two routes of infection. Math. Methods Appl. Sci. (2019). https://doi.org/10.1002/mma.5923

24. Elaiw, A.M., AlShamrani, N.H.: Global properties of nonlinear humoral immunity viral infection models. Int. J. Biomath. 8(5), Article ID 1550058 (2015)

25. Kang, C.J., Miao, H., Chen, X., Xu, J.B., Huang, D.: Global stability of a diffusive and delayed virus dynamics model with Crowley-Martin incidence function and CTL immune response. Adv. Differ. Equ. 2017, 324 (2017)

26. Elaiw, A.M., Alshehaiween, S.F., Hobiny, A.D.: Global properties of delay-distributed HIV dynamics model including impairment of B-cell functions. Mathematics 7, Article ID 837 (2019)

27. Elaiw, A.M., Raezah, A.A., Azoz, S.A.: Stability of delayed HIV dynamics models with two latent reservoirs and immune impairment. Adv. Differ. Equ. 2018, 414 (2018)

28. Gibelli, L., Elaiw, A.M., Alghamdi, M.A., Althiabi, A.M.: Heterogeneous population dynamics of active particles: progression, mutations, and selection dynamics. Math. Models Methods Appl. Sci. 27, 617-640 (2017)

29. Elaiw, A.M., Elnahary, E.Kh.: Analysis of general humoral immunity HIV dynamics model with HAART and distributed delays. Mathematics 7(2), Article ID 157 (2019)

30. Elaiw, A.M., AlShamrani, N.H.: Stability of a general delay-distributed virus dynamics model with multi-staged infected progression and immune response. Math. Methods Appl. Sci. 40(3), 699-719 (2017)

31. Elaiw, A.M., Hassanien, I.A., Azoz, S.A.: Global stability of HIV infection models with intracellular delays. J. Korean Math. Soc. 49(4), 779-794 (2012)

32. Elaiw, A.M., Azoz, S.A.: Global properties of a class of HIV infection models with Beddington-DeAngelis functional response. Math. Methods Appl. Sci. 36, 383-394 (2013)

33. Elaiw, A.M., Abukwaik, R.M., Alzahrani, E.O.: Global properties of a cell mediated immunity in HIV infection model with two classes of target cells and distributed delays. Int. J. Biomath. 7(5). Article ID 1450055 (2014)

34. Bellomo, N., Tao, Y.: Stabilization in a chemotaxis model for virus infection. Discrete Contin. Dyn. Syst., Ser. S 13(2), $105-117(2020)$

35. Wang, Y., Liu, X.: Stability and Hopf bifurcation of a within-host chikungunya virus infection model with two delays. Math. Comput. Simul. 138, 31-48 (2017)

36. Elaiw, A.M., Alade, T.O., Alsulami, S.M.: Analysis of latent CHIKV dynamics models with general incidence rate and time delays. J. Biol. Dyn. 12(1), 700-730 (2018)

37. Elaiw, A.M., Alade, T.O., Alsulami, S.M.: Analysis of within-host CHIKV dynamics models with general incidence rate. Int. J. Biomath. 11(5), Article ID 1850062 (2018)

38. Long, K.M., Heise, M.T.: Protective and pathogenic responses to chikungunya virus infection. Curr. Trop. Med. Rep. 2(1), 13-21 (2015)

39. Wang, J., Lang, J., Zou, X.: Analysis of an age structured HIV infection model with virus-to-cell infection and cell-to-cell transmission. Nonlinear Anal., Real World Appl. 34, 75-96 (2017)

40. Li, F., Wang, J.: Analysis of an HIV infection model with logistic target cell growth and cell-to-cell transmission. Chaos Solitons Fractals 81, 136-145 (2015)

41. Elaiw, A.M., Almatrafi, A., Hobiny, A.D., Hattaf, K.: Global properties of a general latent pathogen dynamics model with delayed pathogenic and cellular infections. Discrete Dyn. Nat. Soc. 2019, Article ID 9585497 (2019)

42. Hobiny, A.D., Elaiw, A.M., Almatrafi, A.: Stability of delayed pathogen dynamics models with latency and two routes of infection. Adv. Differ. Equ. 2018, 276 (2018)

43. Shu, H., Chen, Y., Wang, L.: Impacts of the cell-free and cell-to-cell infection modes on viral dynamics. J. Dyn. Differ. Equ. 30(4), 1817-1836 (2018)

44. Lai, X., Zou, X.: Modeling cell-to-cell spread of HIV-1 with logistic target cell growth. J. Math. Anal. Appl. 426, 563-584 (2015)

45. Hattaf, K., Yousfi, N.: A generalized virus dynamics model with cell-to-cell transmission and cure rate. Adv. Differ. Equ. 2016, $174(2016)$

46. Elaiw, A.M., Raezah, A.A.: Stability of general virus dynamics models with both cellular and viral infections and delays. Math. Methods Appl. Sci. 40(16), 5863-5880 (2017)

47. Lai, X., Zou, X.: Modelling HIV-1 virus dynamics with both virus-to-cell infection and cell-to-cell transmission. SIAM J. Appl. Math. 74, 898-917 (2014)

48. Yang, Y., Zou, L., Ruanc, S.: Global dynamics of a delayed within-host viral infection model with both virus-to-cell and cell-to-cell transmissions. Math. Biosci. 270, 183-191 (2015)

49. Lum, F.M., Ng, L.F.P.: Cellular and molecular mechanisms of chikungunya pathogenesis. Antivir. Res. 120, 165-174 (2015)

50. Korobeinikov, A.: Global properties of basic virus dynamics models. Bull. Math. Biol. 66(4), 879-883 (2004)

51. Huang, G., Takeuchi, Y., Ma, W.: Lyapunov functionals for delay differential equations model of viral infections. SIAM J. Appl. Math. 70(7), 2693-2708 (2010)

52. Shu, H., Wang, L., Watmough, J.: Global stability of a nonlinear viral infection model with infinitely distributed intracellular delays and CTL immune responses. SIAM J. Appl. Math. 73(3), 1280-1302 (2013)

53. Elaiw, A.M.: Global properties of a class of HIV models. Nonlinear Anal., Real World Appl. 11, 2253-2263 (2010)

54. Elaiw, A.M., AlShamrani, N.H.: Global stability of humoral immunity virus dynamics models with nonlinear infection rate and removal. Nonlinear Anal., Real World Appl. 26, 161-190 (2015)

55. Wang, J., Teng, Z., Miao, H.: Global dynamics for discrete-time analog of viral infection model with nonlinear incidence and CTL immune response. Adv. Differ. Equ. 2016, 143 (2016)

56. Elaiw, A.M., AlShamrani, N.H.: Stability of an adaptive immunity pathogen dynamics model with latency and multiple delays. Math. Methods Appl. Sci. 36, 125-142 (2018)

57. Hale, J.K., Lunel, S.V.: Introduction to Functional Differential Equations. Springer, New York (1993)

58. Kuang, Y:. Delay Differential Equations with Applications in Population Dynamics. Academic Press, Boston (1993) 
59. Elaiw, A.M., AlAgha, A.D.: Global dynamics of reaction-diffusion oncolytic M1 virotherapy with immune response. Appl. Math. Comput. 367, Article 124758 (2020)

60. McCluskey, C.C., Yang, Y.: Global stability of a diffusive virus dynamics model with general incidence function and time delay. Nonlinear Anal., Real World Appl. 25, 64-78 (2015)

61. Elaiw, A.M., Alshaikh, M.A.: Stability analysis of a general discrete-time pathogen infection model with humoral immunity. J. Differ. Equ. Appl. (2019). https://doi.org/10.1080/10236198.2019.1662411

62. Xu, J., Hou, J., Geng, Y., Zhang, S.: Dynamic consistent NSFD scheme for a viral infection model with cellular infection and general nonlinear incidence. Adv. Differ. Equ. 2018, 108 (2018)

63. Elaiw, A.M., Alshaikh, M.A.: Stability of discrete-time HIV dynamics models with three categories of infected CD4 ${ }^{+}$ T-cells. Adv. Differ. Equ. 2019, 407 (2019)

Submit your manuscript to a SpringerOpen ${ }^{\circ}$ journal and benefit from:

- Convenient online submission

- Rigorous peer review

- Open access: articles freely available online

- High visibility within the field

- Retaining the copyright to your article

Submit your next manuscript at $\boldsymbol{~ s p r i n g e r o p e n . c o m ~}$ 